\title{
In Vitro and in Vivo Behavior of Liposomes Decorated with PEGs with Different Chemical Features
}

\section{Mastrotto, Francesca}

2020-02

Mastrotto , F , Brazzale , C , Bellato , F , De Martin , S , Grange , G, Mahmoudzadeh , M , Magarkar , A , Bunker , A , Salmaso , S \& Caliceti , P 2020 , ' In Vitro and in Vivo Behavior of Liposomes Decorated with PEGs with Different Chemical Features ' , Molecular Pharmaceutics, vol. 17 , no. 2 , pp. 472-487 . https://doi.org/10.1021/acs.molpharmaceut.9b00887

http://hdl.handle.net/10138/326463

https://doi.org/10.1021/acs.molpharmaceut.9b00887

unspecified

acceptedVersion

Downloaded from Helda, University of Helsinki institutional repository.

This is an electronic reprint of the original article.

This reprint may differ from the original in pagination and typographic detail.

Please cite the original version. 
In vitro and in vivo behavior of liposomes decorated with PEGs with different chemical features.

Francesca Mastrotto $^{1}$, Chiara Brazzale ${ }^{1}$, Federica Bellato ${ }^{1}$, Sara De Martin ${ }^{l}$, Guillaume Grange $^{2}$, Mohamad Mahmoudzadeh ${ }^{2}$, Aniket Magarkar ${ }^{3}$, Alex Bunker $^{2}$, Stefano Salmaso $^{1^{*}}$, Paolo Caliceti ${ }^{1}$

${ }^{1}$ Department of Pharmaceutical and Pharmacological Sciences, University of Padova, via F. Marzolo 5, 35131 Padova - Italy. E-mail stefano.salmaso@unipd.it

${ }^{2}$ Drug Research Program, Faculty of Pharmacy, University of Helsinki, Helsinki - Finland

${ }^{3}$ Institute of Organic Chemistry and Biochemistry, Academy of the Sciences of the Czech Republic, Prague - Czech Republic.

Keywords: stealth liposomes, pharmacokinetic of liposomes, nanocarrier PEG coating.

*Corresponding author

Stefano Salmaso,

Department of Pharmaceutical and Pharmacological Sciences

University of Padova

Via F. Marzolo 5

35131 Padova - Italy

Tel. +390498271602 , Fax. +390498275366

e-mail stefano.salmaso@unipd.it 


\begin{abstract}
The colloidal stability, in vitro toxicity, cell association, and in vivo pharmacokinetic behavior of liposomes decorated with poly(ethylene glycol)-lipids (mPEG-lipids) with different chemical features were comparatively investigated. Structural differences of mPEG-lipids used in the study included: a) surface anchoring moiety [1,2-distearoyl-sn-glycero-3phosphoethanolamine (DSPE), cholesterol (Chol) and cholane (Chln)]; b) mPEG molecular weight, (2 $\mathrm{kDa} \mathrm{mPEG}_{45}$ and $\left.5 \mathrm{kDa} \mathrm{mPEG}_{114}\right)$; $\left.\mathrm{c}\right)$ mPEG shape (linear and branched PEG). In vitro results demonstrated that branched $\left(\mathrm{mPEG}_{114}\right)_{2}$-DSPE confers the highest stealth properties to liposomes ( 31 -fold lower cell association than naked liposomes) with respect to all PEGylating agents tested. However, the pharmacokinetic studies showed that the use of cholesterol as anchoring group yields PEGylated liposomes with longer permeance in the circulation and higher systemic bioavailability among the tested formulations. Liposomes decorated with PEG $_{114}$-Chol had 3.2- and 2.1-fold higher AUC than naked liposomes and branched ( $\left.\mathrm{mPEG}_{114}\right)_{2}$-DSPE coated liposomes, respectively, which reflects the high stability of this coating agent. By comparing the PEGylating agents with same size, namely linear $5 \mathrm{kDa}$ PEG derivatives, linear PPEG $_{114}$-DSPE yielded coated liposomes with the best in vitro stealth performance. Nevertheless, the in vivo the AUC of liposomes decorated with linear $\mathrm{mPEG}_{114-}$ DSPE was lower than that obtained with liposomes decorated with linear $\mathrm{mPEG}_{114}-\mathrm{Chol}$. Computational molecular dynamics modelling provided additional insights that complement the experimental results.
\end{abstract}




\section{INTRODUCTION}

Poly(ethylene glycol) is widely used to endow stealth nanocarriers with prolonged blood circulation. ${ }^{1-3}$ PEGylation can in fact prevent the opsonization of colloidal systems thus minimizing the starvation by macrophages, favor the passive accumulation in tumor or inflamed tissues of the long circulating nanocarriers, reduce systemic toxicity and enhance the therapeutic performance. ${ }^{4}$ Accordingly, liposomes under clinical investigation (e.g. ThermoDox ${ }^{\circledR}$, Lipoplatin ${ }^{\mathrm{TM}}$, S-CKD602, 2B3-101) or already on the market (e.g. Onivyde ${ }$, Doxil®/Caelyx ${ }^{\circledR}$ and LipoDox ${ }^{\circledR)}$ ) boast PEG coating. ${ }^{5}$ However, many studies pointed out that PEG-lipids used to coat liposomes gradually desorb from the liposome surface, resulting in loss of stealth efficiency. PEG detachment from liposome surface is a time- and dilution-dependent phenomenon that may be favored by plasma proteins and shear forces in the blood. ${ }^{6}$ Therefore, PEG-lipids must be stably anchored on liposome surfaces to maintain the biopharmaceutical properties of the vesicles throughout their permeance in the bloodstream.

So far, several investigations reported the effect of the chemical features of PEGylating agents on in vitro/in vivo performance of liposomes. ${ }^{7-10}$ Nevertheless, only a few in vitro and in vivo comparative studies have been performed by using liposomes coated with different PEGylating agents. Therefore, since there is missing information required for the design of performing drug delivery liposomes, their PEGylation is currently based on empiric approaches.

Typically, stealth liposomes are produced by coating the lipid bilayer with monomethoxy-poly (ethylene glycol) (mPEG) conjugated to phospholipids such as 1,2-distearoyl-sn-glycero-3phosphoethanolamine (DSPE) ${ }^{10}$ However, the negative charge of mPEG-phospholipids under physiological conditions may affect their anchoring to the vesicle surface, the liposome pharmacokinetic profiles, and the drug encapsulation efficiency and release and have been demonstrated to activate the complement cascade. ${ }^{11}$ A comparative study of liposomes coated with mPEG-Ceramide, which does not contain phosphate groups, and mPEG-DSPE showed that liposomes coated with the former retain the encapsulated vincristine more efficiently than mPEG-DSPE coated liposomes either in vitro or in vivo ${ }^{10}$ while another study showed that neutral mPEG-lipids minimize the complement activation, ${ }^{11}$ which results in enhanced biopharmaceutical properties.

Although small PEGs are rapidly cleared by kidney ultrafiltration, when anchored onto colloidal platforms they may display a significant toxicity. Indeed, low mol wt PEGs (i.e $\mathrm{Mn}<400 \mathrm{Da}$ ) are more rapidly oxidized by alcohol dehydrogenases than high mol wt PEGs thus producing toxic diacid and hydroxy acid metabolites ${ }^{12}$. On the other hand, PEGs with mol wt 
above the renal clearance cut-off (i.e. $\mathrm{Mn}>30 \mathrm{kDa}$ ) show a low biodegradability and accumulation. ${ }^{13}$

In order to investigate the effect of the chemical and structural features of PEG derivatives on the biopharmaceutical profile of PEG decorated liposomes, we selected branched and linear mPEG derivatives (different architecture) with mPEG mol wt of $2 \mathrm{kDa}$ (45 ethylene glycol monomers) or $5 \mathrm{kDa}$ (114 ethylene glycol monomers) end functionalized with linear DSPE, or polycyclic $5 \beta$-cholanic acid (Chln) or cholesterol (Chol) as surface anchoring groups.

Association of mPEG coated liposomes to cells and their biocompatibility in vitro, and pharmacokinetic studies allowed to correlate the features of the PEGylating agents with the coating stability. Computational molecular dynamics modelling (MD) was applied to obtain mechanistic insights into the effect of the nature of the protective PEG polymer corona on the biological behavior of the drug carrier. Using a model that boasts atomic level resolution, MD provides insight into the system on a length and scale not accessible to experimental techniques. A section of the liposome membrane was modelled for a set of the systems that complements the insight gained regarding the investigated systems to study the interaction of the protective polymer corona with the lipid components of the liposome bilayer, i.e. effect on the overall structure of the liposomes. This study further adds to the insights gained in our past published work, reporting the computational modelling approach applied to study the DOXIL® liposome formulation ${ }^{14}$ and other drug delivery liposome formulations, ${ }^{15-17}$ including other protective polymers than PEG. ${ }^{18}$

Importantly, to the best of our knowledge, no attempts have been made so far to investigate in the same study both the in vitro cell association, biocompatibility and in vivo pharmacokinetic behavior of the liposomal carrier itself. Indeed, information about the polymeric coating performance are usually indirectly obtained by the investigation of the drug payload. Furthermore, most of pharmacokinetic (PK) studies reported in the literature are aimed at investigating the drug fate rather than the carrier, which doesn't provide complete information about the impact that the formulation has in vivo according to its physicochemical features.

\section{MATERIALS AND METHODS}

\subsection{Materials.}

Linear methoxy(polyethylene glycol $2 \mathrm{kDa}$ ) - 1,2-distearoyl-sn-glycero-3-phosphoethanolamine (L-mPEG 45 -DSPE) was purchased from Laysan Bio (Arab, AL, USA); linear methoxy(polyethylene glycol $5 \mathrm{kDa}$ ) - 1,2-distearoyl-sn-glycero-3-phosphoethanolamine (L- 
mPEG$_{114}$-DSPE) was purchased from JenKem Technology (Dallas, Texas, USA); branched [2',3'-Bis(methylpolyoxyethylenoxy)propane-1'-oxycarbonyl $\left.{ }_{5 \mathrm{kDa}}\right]$ - 1,2-distearoyl-sn-glycero3 phosphoethanolamine (B-[mPEG $\left.\left.{ }_{114}\right]_{2}-\mathrm{DSPE}\right)$ was purchased from NOF Europe $\mathrm{GmbH}$ (Frankfurt am Main, Germany). Linear methoxy(polyethylene glycol $5 \mathrm{kDa}$ )-Cholane (L$\mathrm{mPEG}_{114}-\mathrm{Chln}$ ) were synthetized as described by S. Salmaso et al. ${ }^{19,20}$ Sodium chloride $(\mathrm{NaCl})$, ethylenediaminetetraacetic acid (EDTA), Triton X-100 and cholesterol (Chol) were obtained from Sigma-Aldrich (St. Louis, MO, USA). Hydrogenated soy phosphatidylcholine (HSPC) was obtained from Lipoid (Ludwigshafen, Germany) and Rhodamine B 1,2-Dihexadecanoylsn-Glycero-3-Phosphoethanolamine (Rho-DHPE) was purchased from Biotium (Hayward, California, USA). "Ultrapure" water (18.2 M $\Omega$, MilliQ grade) for the preparation of all suspensions and solutions was produced with Millipore MilliQ purification system (MA, USA). All the other chemical reagents and solvents were purchased from Sigma-Aldrich (St. Louis, MO, USA), Baker (Phillipsburg, NJ, USA) and Carlo Erba (Milano, MI, Italia). Hela cells (human epithelial cervix carcinoma cell line) were purchased from the ATCC cell bank (Manassas, VA, U.S.A.). Dulbecco's modified Eagle's medium (DMEM) and RPMI-1640 for cell culture, L-Glutamine solution, fetal bovine serum (FBS), penicillin-streptomycin solution, MEM Non-essential Amino Acid solution, Sodium Pyruvate solution, trypsin, phosphate buffer with and without $\mathrm{CaCl}_{2}$ and $\mathrm{MgCl}_{2}$ (PBS) and 3-[(4,5-dimethylthiazol-2-yl)]-2,5diphenyltetrazolium bromide (MTT), cholesterol hemisuccinate and Lipopolysaccarides from E. coli (LPS) were supplied by Sigma-Aldrich (St. Louis, MO, USA). Human TNF- $\alpha$ DuoSet was obtained from R\&D Systems, Inc. (Minneapolis, MN, USA). Ficoll Paque Plus was purchased from Sigma-Aldrich (St. Louis, MO, USA). 4',6-diamidino-2-phenylindole dihydrochloride (DAPI) and paraformaldehyde (PFA) were provided by Sigma-Aldrich (St. Louis, MO, USA), Wheat Germ Agglutinin AlexaFluor 488 was supplied by Thermo Fisher Scientific (Madison, WI, USA).

Pharmacokinetic studies were carried out by intravenous injection of liposomal formulations on the caudal vein of $36 \mathrm{BALB} / \mathrm{c}$ mice (body weight $20 \pm 2 \mathrm{~g}$, 8-weekold) which were purchased from Charles River (Milan, Italy). Animal care and handling were performed in agreement with the provisions of Italian laws and policies (D.L. ${ }^{\circ} 26$ 14th March 2014) and with the guidelines established by the European Community Council Directive $n^{\circ}$ 2010/63/UE and approved by the veterinary services of the University of Padova (O.P.B.A.-Organismo Preposto al Benessere degli Animali). The in vivo study protocols were approved by the Ethical Committee of the University of Padova and the Italian Ministry of Public Health (approval N ${ }^{\circ}$ 103/2018-PR). 
Pharmacokinetic data were elaborated with the PK Solve, a freely available menu-driven addin program for Microsoft Excel written in Visual Basic for Applications (VBA).

\subsection{Equipment}

Liposomal formulations were extruded with an Avanti Polar Lipids mini-extruder (Alabaster, AL, USA) using a Whatman $0.22 \mu \mathrm{m}$ Nucleopore membrane (Clifton, NJ, USA). Dynamic Light Scattering measurements were performed with Zetasizer NanoZS (Malvern instruments Ltd, UK) equipped with a red $(\lambda=633 \mathrm{~nm})$ laser at a fixed angle of $173^{\circ}$ and DTS applications 6.12 software was used to analyze the data. Particle morphology was analyzed by transmission electron microscopy (TEM) using a Tecnai G2 (FEI, Oregon, USA). Samples were placed on copper grid and allowed to dry at room temperature before the analysis was performed.

Cells were grown using Sanyo $\mathrm{CO}_{2}$ incubator purchased from Panasonic Biomedical Sales (UK) and imaged with optical microscope Axiovert 40CFL Zeiss (Thornwood, NY, USA). Flow cytometric analyses were carried out using a BD FACSCANTO II flow cytometer purchased from BD Biosciences (San Jose, USA) and results were processed with BD FACSDiva Software.

Confocal microscopy images were obtained using a Zeiss LSM 800 confocal laser-scanning microscope (Jena, Germany) equipped with a 63X immersion objective. TEM and confocal images were processed with ImageJ version 1.51f open source Java image software package (National Institutes of Health, USA).

\subsection{Synthesis of methoxy(polyethylene glycol5kDa)-cholesteryl-hemisuccinate (L-mPEG114-} Chol)

Cholesteryl-hemisuccinate $(0.230 \mathrm{~g}, 0.47257 \mathrm{mmol})$ was dissolved in $3 \mathrm{~mL}$ of toluene and the solution was heated to $90{ }^{\circ} \mathrm{C}$. A volume of $343.9 \mu \mathrm{L}$ of thionyl chloride $(4.73 \mathrm{mmol}$ ) weas added. The reaction was stirred under these conditions for 2 hours. The excess of thionyl chloride was removed by distillation and the solution of cholesterol-hemisuccinyl chloride was added to a solution of $100 \mathrm{mg} / \mathrm{mL}$ of $\mathrm{mPEG}_{5 \mathrm{kDa}}-\mathrm{NH}_{2}(0.02 \mathrm{M})$ in toluene containing $75.0 \mu \mathrm{L}$ of triethylamine $(0.54 \mathrm{mmol})$. The reaction was performed at room temperature overnight under stirring. The final product was isolated by precipitation in cold diethyl ether. The product was dialyzed against $\mathrm{mQ}$ water for 48 hours using a 3.5-5.0 kDa MWCO dialysis membrane and lyophilized. The final product was characterized by ${ }^{1} \mathrm{H}-\mathrm{NMR}$, ESI-Tof mass spectrometry and colorimetric assays. The final product yield was $820 \mathrm{mg}(82.0 \% \mathrm{~mol} / \mathrm{mol})$. The conjugation yield was assessed as described above for L-mPEG ${ }_{114}-\mathrm{Chln}$. 
${ }^{1} \mathrm{HNMR}\left(400 \mathrm{mHz}, \mathrm{CDCl}_{3}\right): \delta 0.67\left[\mathrm{~s}, 3 \mathrm{H}, \mathrm{CH}_{3}(\mathrm{C} 19)\right.$ of cholesterol], 1.01 [s, 6H, $\left(\mathrm{CH}_{3}\right)_{2}(\mathrm{C} 26-$ C27) of cholesterol], 3.38 [s, 3H, $\mathrm{CH}_{3} \mathrm{O}-\mathrm{PEG}$ ], $\delta 3.64$ [s, $4 \mathrm{nH},-\left(\mathrm{CH}_{2} \mathrm{CH}_{2}-\mathrm{O}\right)_{\mathrm{n}}$ - of PEG].

ESI-Tof: bell shape profile centered at $2700[\mathrm{M}+2 \mathrm{H}]^{2+}$, [calcd MW for $\mathrm{mPEG}_{5 \mathrm{kDa}-\text { cholesterol }}$ 5403].

\subsection{Preparation of liposomes}

Naked liposomes were prepared by the thin-film hydration method ${ }^{21}$ using a 2:1 hydrogenated soybean phosphatidylcholine/cholesterol (HSPC/Chol) molar ratio. Briefly, $6.67 \mathrm{mg} / \mathrm{mL}$ of HSPC and Chol stock solutions in chloroform $\left(\mathrm{CHCl}_{3}\right)$ were prepared. 1,202 $\mu \mathrm{L}$ of HSPC $(8.02$ $\mathrm{mg}, 10.23 \mu \mathrm{mol})$ and $296 \mu \mathrm{L}$ of Chol (1.98 mg, $5.12 \mu \mathrm{mol})$ from stock solutions were mixed. The organic solvent was removed under reduced pressure and the obtained lipid film was rehydrated with $200 \mu \mathrm{L}$ of $10 \mathrm{mM}$ phosphate buffer, $140 \mathrm{mM} \mathrm{NaCl}$ (PBS, pH 7.4 unless otherwise indicated) under vigorous stirring and processed with ten freeze-thaw cycles. The concentrated lipid dispersion $(50 \mathrm{mg} / \mathrm{mL})$ was diluted to a final concentration of $10 \mathrm{mg} / \mathrm{mL}$ with PBS, sonicated for 10 minutes and finally extruded eleven times through a $200 \mathrm{~nm}$ cut-off polycarbonate membrane to obtain small unilamellar vesicles.

Cationic liposomes were prepared as described above by including 1,2-dioleoyl-3trimethylammonium-propane (DOTAP) in the lipid mixture. Briefly, HSPC (8.02 mg, 10.23 $\mu \mathrm{mol}, 1,202 \mu \mathrm{L})$ and Chol $(1.98 \mathrm{mg}, 5.12 \mu \mathrm{mol}, 297 \mu \mathrm{L})$ solutions in chloroform were mixed with a DOTAP solution in ethanol (4.61 mg, $6.63 \mu \mathrm{mol}, 92 \mu \mathrm{L})$ at a molar ratio of 46.7:23.3:30, respectively. Liposomes were processed as described above to obtain a final lipid concentration of $10 \mathrm{mg} / \mathrm{mL}$.

PEGylated liposomes were obtained by post-insertion method from $50 \mathrm{mg} / \mathrm{mL}$ naked liposomes.

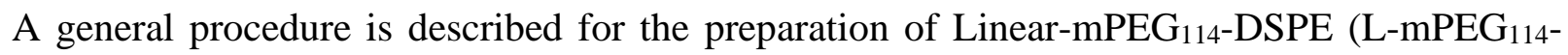
DSPE) coated liposomes. A $5 \mathrm{mg} / \mathrm{mL}$ L-mPEG 114 -DSPE solution was prepared in PBS. A volume of $779 \mu \mathrm{L}$ of this solution $(4.67 \mathrm{mg}, 0.81 \mu \mathrm{mol})$ was gradually added to the concentrated lipid dispersion providing concentrations of $\mathrm{L}_{-} \mathrm{mPEG}_{114}-\mathrm{DSPE}$ above its $\mathrm{CMC}^{22}$ (Table S1). The mixture was sonicated at $37{ }^{\circ} \mathrm{C}$ for 1 minute using a tip sonicator Omni - Ruptor 250 Ultrasonic Homogenizer (OMNI International Inc., Kennesaw, GA -USA) at 20\% of the maximum power. The sample was then diluted with PBS at $\mathrm{pH} 7.4$ to obtain a final lipid concentration of $10 \mathrm{mg} / \mathrm{mL}$ and a HSPC:Chol:PEG ratio of 63.3:31.7:5 (mol:mol:mol). Aliquots of $1 \mathrm{~mL}$ of each liposome formulation was centrifuged for $30 \mathrm{~min}$ at 14,000 rpm (Sigma 1-14 microfuge, Newtown, UK) to isolate the liposomal pellet. Then, the supernatants were recovered, and PEG concentration 
was tested by Iodine assay ${ }^{23}$. Supernatants were also analysed for lipid concentration by Stewart assay to evaluate the efficiency of liposome isolation by centrifugation. ${ }^{24}$

All the other liposomal formulations were prepared by varying the coating agent - i.e. LmPEG$_{45}$-DSPE, L-mPEG $114-$ Chln, L-mPEG $114-$ Chol and Branched-mPEG $114-\mathrm{DSPE}$ (B$\left.\left[\mathrm{mPEG}_{114}\right]_{2}-\mathrm{DSPE}\right)$. In the case of B-[mPEG$\left.{ }_{114}\right]_{2}-\mathrm{DSPE}$, the HSPC:Chol:B-[mPEG $\left.{ }_{114}\right]_{2}-\mathrm{DSPE}$ ratio was 65:32.5:2.5 (mol:mol:mol) to provide the same PEG chain density as compared to the other formulations containing linear PEG. The concentration of all PEG-lipid agents during the post-insertion procedure was above their CMC (Table S1).

All liposome preparations were performed under controlled conditions by using sterile buffer, sterilized glassware, sterile plastics, and under a Biosafety Cabinet. The liposomal formulations were tested immediately after preparation to avoid the risk of contamination.

\subsection{Size, morphology and stability}

One $\mathrm{mg} / \mathrm{mL}$ liposomal formulations in PBS were characterized by Dynamic Light Scattering (DLS) at $25{ }^{\circ} \mathrm{C}$ to assess vesicle mean size, polydispersity index (PDI) and zeta potential $(\zeta$ potential). Morphological analysis of liposomal formulations in PBS was performed by Transmission Electron Microscopy (TEM) at a concentration of $0.3 \mathrm{mg} / \mathrm{mL}$. Samples were deposited on a carbon coated copper grid and the solvent was allowed to dry at room temperature. The excess volume was removed using filter paper; samples were negatively stained with $1 \%$ (w/v) uranyl acetate in distilled water and imaged.

To investigate the stability of liposomes in serum, liposomal formulations were diluted 10 times with PBS containing $2.8 \% \mathrm{v} / \mathrm{v}$ foetal bovine serum (FBS) to a final lipid concentration of 1 $\mathrm{mg} / \mathrm{mL}$ and $2.5 \% \mathrm{v} / \mathrm{v}$ FBS. The formulations were then characterized in terms of size (expressed as intensity) and polydispersity by DLS at scheduled times (0, 0.5, 1, 2, 4, 8, 24 hours after sample dilution). Three independent experiments were performed in triplicate.

\subsection{Fluorescent labelling and validation of liposome quantification in biological media}

The in vitro and in vivo biological studies were performed by labelling liposomal formulations with the fluorescent probe Rhodamine B-1,2-diesadecanoil-sn-glycero-3-phosphoethanolamine (Rho-DHPE). $0.5 \mathrm{~mol} \%$ of Rho-DSPE was added to the initial lipid mixture in organic solvent and then the same procedure described above was used for liposome preparation. From now on, labelled liposomes will be referred to as "Rho-liposomes". 
Fluorescent labelling stability in presence of serum proteins: Rho-liposomes were diluted ten times with FBS to a lipid concentration of $1 \mathrm{mg} / \mathrm{mL}(500 \mu \mathrm{L})$ and $2.5 \% \mathrm{v} / \mathrm{v}$ FBS and incubated for 24 hours at room temperature. The mixtures were then eluted through a chromatographic column prepacked with Sepharose CL-4B medium using PBS as mobile phase. Fractions of 1 $\mathrm{mL}$ were collected and analyzed by UV-Vis spectrophotometry at $280 \mathrm{~nm}$ and $552 \mathrm{~nm}$. Control experiments were performed by eluting separately Rho-liposomes and FBS under the same conditions to identify their elution profile and time of elution.

Rho-liposome recovery from different media: $10 \mu \mathrm{L}$ of Rho-liposomes in PBS at increasing concentration were diluted with $30 \mu \mathrm{L}$ of PBS, or plasma or murine blood in order to have a final concentration of Rho-DHPE from 0 to $140 \mathrm{ng} / \mathrm{mL}$. The mixtures were incubated at room temperature for 24 hours. $20 \mu \mathrm{L}$ of the mixtures in PBS and in plasma were added to $500 \mu \mathrm{L}$ of methanol and centrifuged at 12,000 rpm for 4 minutes to remove the plasma proteins. The mixtures in murine blood underwent an additional centrifugation step (1,500 rpm for 10 minutes) to isolate erythrocytes first and then the plasma was mixed with methanol to remove plasma proteins as reported above. Fluorescence emission intensities of the supernatants were recorded at $\lambda_{\mathrm{em}} 580 \mathrm{~nm}$ with excitation wavelength at $560 \mathrm{~nm}$ and plotted versus Rho-DHPE concentration.

\subsection{In vitro studies}

\subsubsection{Hemolysis}

Erythrocytes (RBCs) were isolated from heparinized murine blood by centrifugation at 1,100 rpm for 5 minutes at $4{ }^{\circ} \mathrm{C}$. The pellet was washed thrice with sterile saline solution $(140 \mathrm{mM}$ $\mathrm{NaCl}$ in MilliQ water) and resuspended in PBS to yield a $2 \% \mathrm{w} / \mathrm{w}$ haematocrit concentration (2 $\mathrm{mL}$ ). Afterwards, volumes of $190 \mu \mathrm{L}$ of RBCs suspensions were mixed with $10 \mu \mathrm{L}$ of liposomal formulations in PBS at the following lipid concentrations: 1.0, 2.0, 6.0 and $10 \mathrm{mg} / \mathrm{mL}$. Samples were prepared in sixplicate. The samples were incubated at $37{ }^{\circ} \mathrm{C}$ for 1 hour and then centrifuged at 1,100 rpm for 5 minutes to remove unlysed RBCs. Supernatants were collected and the absorption of the released haemoglobin was measured by spectroscopic analysis at 570 $\mathrm{nm}$ using an ELISA plate reader. The haemolytic activity of liposomes was plotted as a percentage of hemoglobin released with respect to RBCs samples treated with $1 \%$ (w/v) Triton $\mathrm{X}-100$ which induces complete lysis (100\%), as indicated in equation. RBCs incubated with PBS were used as negative control. ${ }^{25}$ 


\subsubsection{Cell culture}

Hela cells (human epithelial cervix carcinoma) were cultured in monolayer in Dulbecco's modified Eagle's medium (DMEM) supplemented with 10\% (v/v) FBS, 2.0 mM L-glutamine, $100.0 \mathrm{IU} / \mathrm{mL}$ penicillin, $100.0 \mu \mathrm{g} / \mathrm{mL}$ streptomycin and $0.25 \mu \mathrm{g} / \mathrm{mL}$ amphotericin B (complete DMEM) in a humidified atmosphere containing $5 \% \mathrm{CO}_{2}$ at $37^{\circ} \mathrm{C}$.

\subsubsection{Cytotoxicity studies.}

Hela cells were seeded in 96-well plates $\left(1 \times 10^{4}\right.$ cells/well) and grown for 24 hours. The medium was replaced with $200 \mu \mathrm{L}$ of a $1 \mathrm{mg} / \mathrm{mL}$ liposomal suspension in DMEM added of $10 \% \mathrm{FBS}$ and incubated for 2, 4, 6 and 24 hours. Afterwards, the medium containing liposomes was removed and cells were washed twice with PBS $(100 \mu \mathrm{L})$ and $200 \mu \mathrm{L}$ of complete DMEM was added to each well. Cell viability was assessed by MTT assay. ${ }^{26} 20 \mu \mathrm{L}$ of a $5 \mathrm{mg} / \mathrm{mL}$ MTT solution in PBS was added to each well and the plates were incubated for 3 hours at $37^{\circ} \mathrm{C}$. The medium was then removed and $200 \mu \mathrm{L}$ of DMSO was added to each well to dissolve the formazane crystals. The plates were gently shaken for 15 minutes and absorbance was measured at $570 \mathrm{~nm}$ using ELISA plate reader. Untreated cells were used as positive control (100\% cell viability).

\subsubsection{Activation of inflammation study}

Leukocyte-enriched blood (buffy coat) from healthy human donors was obtained from the Blood Bank of the Hospital of Padova and supplemented of EDTA (1.8 mg per mL of blood). One blood unit $(\sim 50-60 \mathrm{~mL})$ was diluted to a final volume of $215 \mathrm{~mL}$ with isolation buffer (PBS enriched with $2 \mathrm{mM}$ EDTA, pH 7.2). $35 \mathrm{~mL}$ aliquots of diluted blood was carefully and slowly layered over $15 \mathrm{~mL}$ of Ficoll Paque Plus in a $50 \mathrm{~mL}$ conical tube. Then, tubes were centrifuged at $400 \mathrm{x}$ g for 40 minutes at $20{ }^{\circ} \mathrm{C}$ in a swinging-bucket rotor without brake. The upper layer of plasma was aspirated leaving the mononuclear cell (PBMCs) layers undisturbed at the interphase. Three PBMCs layers were collected into a new $50 \mathrm{~mL}$ conical tube, diluted to a final volume of $50 \mathrm{~mL}$ with isolation buffer, mixed and centrifuged again at $300 \mathrm{x} \mathrm{g}$ for 10 minutes at $20{ }^{\circ} \mathrm{C}$ in a swinging-bucket rotor without brake. The supernatant was completely removed, the cell pellet was resuspended in $50 \mathrm{~mL}$ of isolation buffer and centrifuged at $200 \mathrm{x}$ g for 10 minutes at $20{ }^{\circ} \mathrm{C}$ to ensure the complete removal of platelets. The supernatant was carefully removed and the PBMCs pellet was resuspended with $30 \mathrm{~mL}$ PBMCs Growth Medium (RPMI supplemented with 10\% FBS, 2 mM L-Glutamine, 1 mM Sodium Pyruvate, 1\% non-essential amino acids, 100 $\mathrm{U} / \mathrm{mL}$ Penicillin and $100 \mu \mathrm{g} / \mathrm{mL}$ Streptomycin) and manually counted by using a Bürker chamber. 
Freshly isolated PBMCs were seeded into a 12-well plate at a density of $3 \times 10^{6}$ cells $/ \mathrm{mL}$ in PBMCs Growth Medium (900 $\mu \mathrm{L}$ per well) and added of $100 \mu \mathrm{L}$ of $10 \mathrm{mg} / \mathrm{mL}$ liposomal formulations suspended in PBS. Cells added with PBS (100 $\mu \mathrm{L} /$ well) or a $40 \mu \mathrm{g} / \mathrm{mL}$ solution of lipopolysaccharides (LPS) from E. coli $(100 \mu \mathrm{L} /$ well) in PBS were used as negative and positive control, respectively. After $16 \mathrm{~h}$ of incubation, cells were transferred into conical vials, centrifuged at $800 \mathrm{~g}$ for $7 \mathrm{~min}$ and the supernatants were collected. The concentrations of TNF$\alpha$ in cell culture supernatants were quantified by Human DuoSet ELISA kits obtained from R\&D Systems according to the manufacturer's instructions.

\subsubsection{Liposome association with cells.}

Cytofluorimetry. Hela cells were seeded in 24 -well plates $\left(7 \times 10^{4}\right.$ cells/well) in complete DMEM medium and grown at $37{ }^{\circ} \mathrm{C}$ for 24 hours. Then, the medium was removed and the cells were treated with $500 \mu \mathrm{L}$ of $1 \mathrm{mg} / \mathrm{mL}$ Rho-liposomes in complete DMEM. Cells were incubated with liposomal formulations at $37{ }^{\circ} \mathrm{C}$ for $1,2,4,6$ and 24 hours. Afterwards, the medium was discharged and cells were washed thrice with PBS $(500 \mu \mathrm{L} /$ well $)$ and detached by treatment with $500 \mu \mathrm{g} / \mathrm{mL}$ trypsin in PBS $\left(200 \mu \mathrm{L} /\right.$ well). After 4 minutes of incubation at $37{ }^{\circ} \mathrm{C}$, the enzyme activity was quenched by addition of $600 \mu \mathrm{L}$ of complete DMEM. Cell suspensions were transferred into flow cytometry tubes and centrifuged at 1,000 rpm for 5 minutes. The supernatant was discharged and the cellular pellet was resuspended in $300 \mu \mathrm{L}$ of PBS and analysed by flow cytometer BD FACSCANTOII (488-nm blue laser). The data were elaborated using BD FACSDiva software. Three independent experiments were performed in triplicate.

Confocal microscopy. Hela cells were seeded onto imaging dishes $\left(5 \times 10^{4}\right.$ cells/well $)$ in complete DMEM and grown at $37{ }^{\circ} \mathrm{C}$ in a humidified atmosphere containing $5 \% \mathrm{CO}_{2}$. After 24 hours, the medium was discharged and replaced with $1 \mathrm{mg} / \mathrm{mL}$ Rho-liposomes in complete DMEM (500 $\mu \mathrm{L} /$ well). The plates were incubated at $37^{\circ} \mathrm{C}$ for 6 hours. Then, cells were washed thrice with PBS (500 $\mu \mathrm{L} /$ well) and fixed with $300 \mu \mathrm{L}$ of $4 \%$ (w/v) paraformaldehyde solution in PBS (500 $\mu \mathrm{L} /$ well) for 15 minutes at room temperature. Cells were washed three times with PBS and incubated with $300 \mu \mathrm{L}$ of a $5 \mu \mathrm{g} / \mathrm{mL}$ Wheat Germ Agglutinin (WGA)-AlexaFluor® 488 conjugate solution in PBS for 15 minutes at room temperature. Afterwards, cells were washed three times with PBS (500 $\mu \mathrm{L} /$ well) and incubated with $300 \mu \mathrm{L}$ of a $4.5 \mu \mathrm{g} / \mathrm{mL}$ DAPI solution in PBS for 15 minutes at room temperature. Cells were then washed thrice with PBS. Slides were mounted on microscope slides and confocal microscopy images were acquired with a Zeiss LSM 800 confocal laser-scanning microscope equipped with a $63 \mathrm{X}$ oil immersion objective lens. 
Laser irradiation at $408,488,561 \mathrm{~nm}$ was used to detect DAPI, WGA-AlexaFluor ${ }^{\circledR} 488$ and Rho-DHPE, respectively. Images elaboration was performed using ImageJ Software version $1.51 \mathrm{j} 8$.

\subsection{Pharmacokinetic study}

$\mathrm{BALB} / \mathrm{c}$ mice were randomly divided into 6 groups each of 6 mice and housed at the Department of Pharmaceutical and Pharmacological Sciences of the University of Padova under conditions of $25{ }^{\circ} \mathrm{C}$ and $50 \%$ humidity, with free access to standard food and water. Mice were intravenously injected via the tail vein with $200 \mu \mathrm{L}$ of $10.0 \mathrm{mg} / \mathrm{mL}$ liposome suspension. At scheduled times $(0.02,0.5,1,2,4,6$ and 24 hours) $100 \mu \mathrm{L}$ of blood sample were withdrawn by submandibular venous plexus puncture of anesthetized mice and centrifuged at 2,500 rpm for 6 minutes. Plasma samples $(20 \mu \mathrm{L})$ were added of $500 \mu \mathrm{L}$ of methanol. Each sample was then centrifuged at 12,000 rpm for 5 minutes and the supernatant was analysed by spectrofluorometer. Liposome concentration in the supernatants was derived from Rho-DSPE fluorescence using a calibration curve obtained analyzing dilutions of Rho-DHPE in methanol $(y=11.254 x+19.967$, $\mathrm{R}^{2}$ 0.997). The pharmacokinetic parameters of liposomal formulations following intravenous administration were calculated using PK solver 2.0 software.

\subsection{Statistical analysis}

All samples were prepared in triplicate and data are shown as mean \pm standard deviation of three independent experiments. Statistical analysis was performed using Graph-Pad Prism 6.0 software. Statistical significance was calculated using Bonferroni test and one-way analysis of variance (ANOVA), setting $\mathrm{p}<0.05$ as statistical significance.

\subsection{Computational study}

Computational molecular dynamics modelling (MD) was used to study a set of 7 liposome membrane models, a set of systems designed to provide insight that complements the results obtained from the experimentally studied systems. The MD simulations involved modelling the structure and behavior of a section of the lipid membrane, as a model for the surface properties of the liposome, and structure and stability of the liposome membrane.

In all 7 cases the membrane in the model was composed of DSPC and Cholesterol, with molar ratio set to match the experimental systems (Table S2 in Supplementary Information). The systems modelled the range of variations regarding PEGylation that we studied experimentally; 
this includes varying the length of the PEG polymer, its structure and altering the lipid with which the PEG is functionalized. Specifically, we studied PEG lengths $2 \mathrm{kDa}\left(\mathrm{PEG}_{45}\right)$ and 5 $\mathrm{kDa}\left(\mathrm{PEG}_{114}\right)$, considered linear and branched $\mathrm{PEG}_{114}$ and considered the case of $\mathrm{mPEG}$ functionalized with DSPC (transforming it to mPEG-DSPE), cholesterol (Chol) and cholane (Chln).

The 7 systems modelled, shown in Table S2 in Supplementary Information, are: 1) $\mathrm{mPEG}_{45}$ DSPE, corresponding to a section of the membrane of a commercial DOXIL ${ }^{\circledR}$ liposome formulation ${ }^{27}$ that has been studied using our computational toolkit in a previous publication, ${ }^{14}$

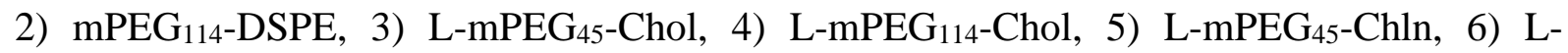
$\mathrm{mPEG}_{114}-\mathrm{Chln}$ and 7) B-[mPEG$\left.{ }_{114}\right]_{2}-\mathrm{DSPE}$.

For the systems with $\mathrm{PEG}_{114}$, both linear and branched, we only included PEGylated molecules on one leaf of the membrane due to the enlarged size of the simulation box required, while, for all systems with $\mathrm{PEG}_{45}$, we considered PEGylated molecules in both leafs of the membrane, as we have performed in our previous simulations of polymer functionalized membranes. ${ }^{14,18,28-}$ ${ }^{32}$ In our simulations we did not study the distribution of PEGylated molecules between the two leaves of the membranes as this property of the liposomes cannot be studied using MD simulation with all atom resolution; each leaf of the membrane with PEGylated molecules represents a simulation of the outer leaf of the liposome membrane. In all simulated systems we included salt ions, to approximate physiological salt concentration $(\sim 125 \mathrm{mM} \mathrm{NaCl})$.

All simulations were carried out using the GroMACS 5.1.5 open source software suite. ${ }^{33,34}$ All membrane systems were simulated using the Optimized Potentials for Liquid Simulations - All Atom (OPLS-AA) force field parameter set ${ }^{35,36}$ and all lipids were modelled using the MacRog lipid potential set. ${ }^{37}$ Further details regarding the construction of the molecular force fields, other simulation parameters and initialization procedures, are found in the Methods of Supplementary Information. All systems were built using software developed in-house with composition shown in Table S2 of Supplementary Information. Once the initialization procedure described in the supplementary materials was completed, the PEGylated membranes were all simulated for a total molecular dynamics trajectory of $800 \mathrm{~ns}$. The portion of this trajectory required for relaxation of the systems (i.e. losing the memory of the starting configuration and reaching equilibrium) was determined through calculation of both the percentage of $\mathrm{Na}^{+}$ions in contact with the PEG oxygens and DSPC lipid headgroups and the area per lipid as a function of time, to be $\sim 450 \mathrm{~ns}$; all analysis regarding the PEGylated membranes simulated was made using the latter $350 \mathrm{~ns}$ of the MD trajectory. For system 1, that corresponds to the DOXIL ${ }^{\circledR}$ formulation, ${ }^{27}$ we show the same analysis made from the 
equilibrated trajectory from our previous published work. We simulated all systems for a total of $800 \mathrm{~ns}$ and thus performed our analysis on the last $350 \mathrm{~ns}$ of the simulation trajectories. For system 1, the results are taken from the portion of the trajectory analyzed in our previous study. ${ }^{14}$

In analyzing the trajectories, in addition to the $\mathrm{Na}^{+}$ion contacts and area per lipid, used to determine the time needed for the systems to relax, we calculated four additional properties of the lipid membranes: 1) the deuterium order parameters for the two lipid acyl chains (namely Sn-1 and Sn-2) and 2) the charge density, 3) electrostatic potential and 4) mass density profile of the components of the system, along the membrane normal, i.e. perpendicular to the membrane surface. The deuterium order parameter $\left(\mathrm{S}_{\mathrm{CD}}\right)^{38}$ is a property of the lipid bilayer that can be derived accurately from NMR experiments, in addition to MD simulation of the model of the system; it is an extremely useful gauge of the level of lipid chain ordering. It is defined as:

$$
S_{C D}=\left\langle\frac{3}{2} \cos ^{2} \theta_{i}-\frac{1}{2}\right\rangle
$$

where $\theta_{i}$ is the angle between the $\mathrm{C}-\mathrm{D}$ bond $(\mathrm{C}-\mathrm{H}$ in simulations) of the $\mathrm{i}$-th carbon atom and the membrane normal. The angular brackets denote averaging over time and over relevant $\mathrm{C}-\mathrm{D}$ bonds in the bilayer. The electrostatic potential calculation involves first calculating the charge density along the membrane normal, then integrating this result twice. The mass density profile is a measure of the relative densities of different elements of the system along the membrane normal; it serves as a measure of where in the membrane different atoms, molecules or molecule groups sit. All calculations of the deuterium order parameter, electrostatic potential and mass density profile were made using the MD trajectory analysis tools available through the GroMACS 5.1.5 open source software suite. ${ }^{33,34}$ Additionally, we visualized the simulated systems using the Visual Molecular Dynamics (VMD) open source software package. ${ }^{39}$

\section{RESULTS AND DISCUSSION}

A variety of mPEG-lipids are available for liposome coating. ${ }^{40}$ However, the liposome stability and shielding efficiency of the PEGylating agents depend on the chemical and structural features of the PEGylaying agents, namely anchoring group and PEG moiety.

In this comparative study the effect of molecular weight, architecture and terminal anchoring function of PEGylating agents on the biological performances of liposomes was investigated 
by using PEG-lipids formed by mPEG chains with different mol wt (2-10 kDa) and structure (linear or branched) and by linear or polycyclic lipophilic anchoring groups (DSPE, cholane or cholesterol, Figure 1).

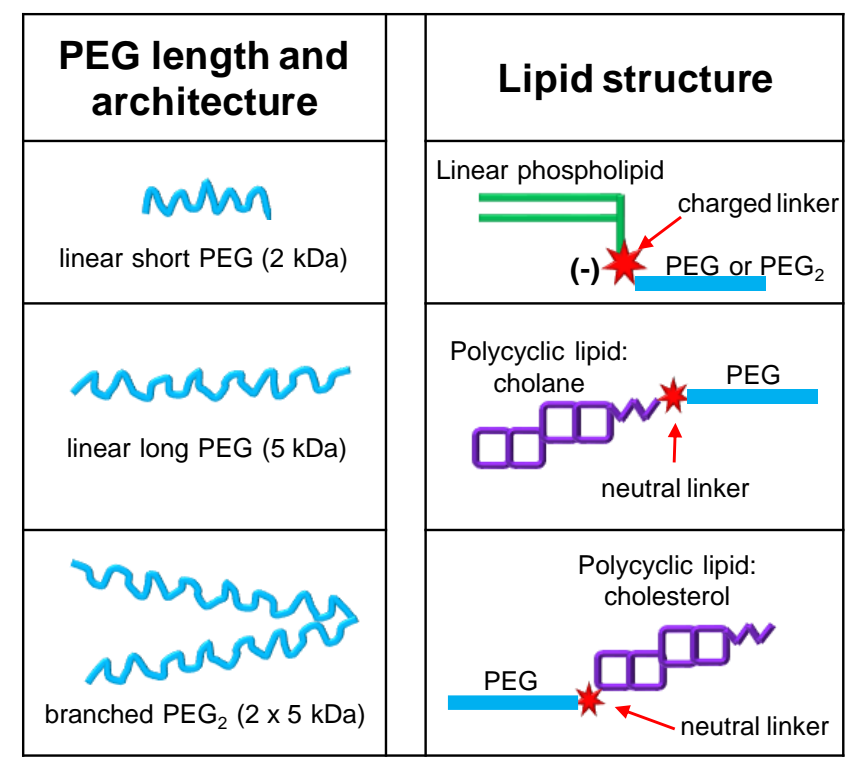

Figure 1. Schematic representation of PEGylating agent components investigated.

Phospholipids, e.g. DSPE, are the classical anchoring lipids used for liposome PEGylation. As an example, Doxil@, a commercially available liposomal doxorubicin formulation, contains $\mathrm{mPEG}_{2 \mathrm{kDa}}-\mathrm{DSPE}$ as PEGylating agent where $\mathrm{mPEG}-\mathrm{OH}$ is chemically conjugated to the terminal amino group of the lipid head yielding a -HN-COO- urethane linkage. ${ }^{41}$

Cholesterol (27-carbon steroid) possesses an $-\mathrm{OH}$ function in position 3 of the polycyclic structure, while $5 \beta$-cholanic acid (saturated 24-carbon steroid) contains one- $\mathrm{COOH}$ in position 17 of the alkyl moiety of the steroid structure. These functions can be properly activated for conjugation to mPEG- $\mathrm{NH}_{2}$ resulting in $\mathrm{mPEG}-\mathrm{Chol}$ where the polymer is linked to $\mathrm{C} 3$ of the polycyclic moiety while in the mPEG-Chln the polymer is conjugated to $\mathrm{C} 17$ of the alkyl chain of the cholane moiety. Therefore, despite the hydrophobic moieties at the PEG chain have similar structure, they have different spatial orientation in the two derivatives. ${ }^{19}$ Therefore, although by virtue of their lipophilicity and structural similarity both Chol and Chln can be incorporated into the liposomal bilayer, their different orientation can yield different interaction with the lipids of liposome bilayer. Furthermore, also mPEG may control their interaction with the liposome bilayer, which in turn can affect the stability of the polymeric coating ${ }^{42}$, and the biopharmaceutical properties of the vesicles. mPEG functionalized with $5 \beta$-cholanic acid has 
been investigated here for the first time as PEGylating agent for liposome coating while afew studies have been performed with mPEG-Chol derivatives. ${ }^{43}$

Several studies have shown that cholesterol, by virtue of its condensing and a fluidizing effect ${ }^{44}$, 45 of the liquid-crystalline membrane of liposomes decreases the bilayer permeability and improves the retention of hydrophilic drug payload. ${ }^{46}$ Therefore, the two polycyclic lipids attached to mPEG are expected to contribute in a different way to the bilayer stabilization.

Aside the length and architecture of mPEG chains, polymer density and the polymer chain conformation on liposome surface strongly affect the stealth properties of liposomes. ${ }^{47,48}$ The opsonization of liposomes decreases upon transition of the PEG coating from flattened "mushroom"- to extended -"brush"- conformation, which occurs by increasing the polymer chain density on the liposome surface. ${ }^{49}, 50$ Indeed, computational simulations carried out by Lee et al. demonstrated that $\mathrm{PEG}_{17}$ with low $\mathrm{Mn}$ (e.g. $\left.750 \mathrm{Da}\right)$ is in the "mushroom-like" state regardless the polymer surface density. Other studies showed that the decoration of liposomes with $2 \mathrm{kDa} \mathrm{PEG} 45$ at a density of $5 \mathrm{~mol}^{51}$ or with $5 \mathrm{kDa} \mathrm{PEG}_{114}$ at a density of 2-6.3 mol \% ${ }^{52}$ reduces liposome aggregation and inhibit the binding of plasma proteins to liposomes as a result of a predominant "brush-like" conformation. ${ }^{49}$ In light of these evidences, we performed a comparative study by decorating liposome with two different linear mPEGs $\left(2 \mathrm{kDa}^{\mathrm{mPEG}} 45\right.$ and $5 \mathrm{kDa} \mathrm{mPEG}_{114}$ ) and surface polymer density of $5 \mathrm{~mol}_{\%} . \mathrm{mPEG}_{45}$ has been exploited for the surface coating of commercial liposomes (e.g.: Doxil ${ }^{\circledR}$, Caelyx) while $\mathrm{mPEG}_{114}$ is used for the PEGylation of several commercial drugs (e.g. Oncospar, Somavert, Adagen). Longer mPEG chain derivatives combine with phosphatidylcholine to form phase separated lamellae, making them unsuitable for the preparation of long circulating liposomes. ${ }^{53}$ Branched $\left(\mathrm{mPEG}_{114}\right)_{2}$ derivative at surface polymer density of $5 \mathrm{~mol} \%$ was used to investigate the effect of the architecture.

The structures of the mPEG-lipid conjugates used in the present comparative investigation for liposome coating are reported in Figure 2. 
A

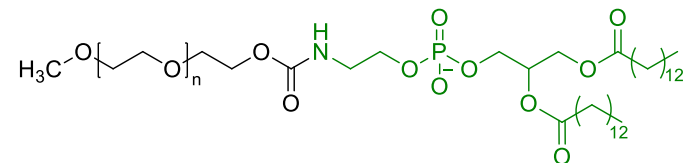

L-mPEGn-DSPE

$A^{\prime} \quad n=45 \quad M n 2 \mathrm{kDa}$

$A^{\prime \prime} \mathrm{n}=114 \quad \mathrm{Mn} 5 \mathrm{kDa}$

C

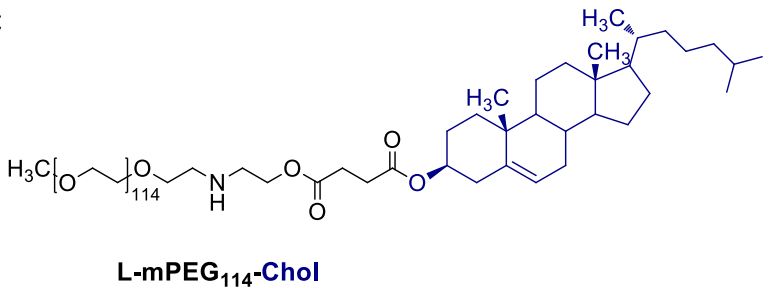

B

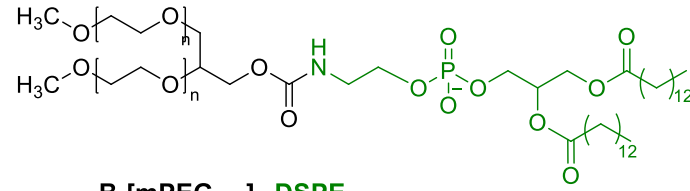

B-[mPEG $\left.{ }_{114}\right]_{2}-\mathrm{DSPE}$

D

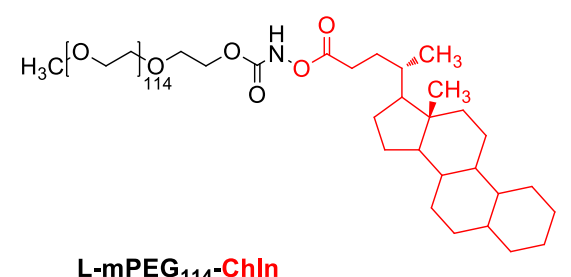

Figure 2. Chemical structure of mPEG-lipid derivatives used for liposome coating. A'. Linear-

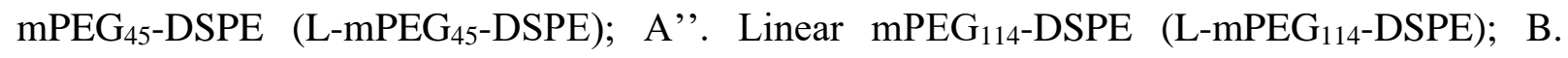
Branched $\mathrm{mPEG}_{114}$-DSPE [B-(mPEG$\left.{ }_{114}\right)_{2}$-DSPE]; C. Linear mPEG ${ }_{114}$-Cholesterol (L-mPEG ${ }_{114}$ Chol); D. Linear mPEG $114-$ Cholane (L-mPEG $114-\mathrm{Chln})$.

\subsection{Formulation of PEGylated liposomes}

The analysis performed after liposome PEGylation showed that the procedure yielded over 95\% post-insertion efficiency for all PEG-lipid agents. The size and surface charge of coated liposomes, which are the main features dictating their biopharmaceutical and pharmacokinetic behavior, were determined by dynamic light scattering (DLS) and transmission electron microscopy (TEM). ${ }^{54}$

The DLS profiles reported in Figure 3A and the data reported in Table I show that all PEGylated liposome formulations possess a mean diameter in the range of 165-180 nm and narrow polydispersity $(\mathrm{PDI}<0.2)$, which are compatible with their passive diffusion across the endothelial fenestrae of the tumor vessels and accumulation in the tumor tissue by enhanced permeation and retention (EPR) effect. ${ }^{55,56}$ Naked and cationic liposomes displayed larger sizes compared to PEGylated liposomes. Naked liposomes showed high PDI, which indicates a broad size distribution. 
Table I. Size (Intensity), polydispersity index (PDI) and zeta potential of naked liposomes, cationic liposomes, and liposomes coated with selected PEGylating agents: L-mPEG ${ }_{45}-\mathrm{DSPE}$, L-mPEG ${ }_{114}$-DSPE, B-(mPEG 114$)_{2}$-DSPE, L-mPEG ${ }_{114}-C h l n$, L-mPEG $_{114}$-Chol.

\begin{tabular}{|c|c|c|c|}
\hline Rho-liposomes $^{\text {a }}$ & Dimensions (d.nm) & PDI & $\zeta$-potential $(\mathrm{mV})$ \\
\hline Naked & $226 \pm 32.7$ & $0.48 \pm 0.13$ & $-0.22 \pm 0.51$ \\
\hline Cationic liposomes & $237 \pm 4.62$ & $0.10 \pm 0.081$ & $13.2 \pm 0.21$ \\
\hline L-mPEG $45-\mathrm{DSPE}$ & $167 \pm 5.17$ & $0.17 \pm 0.11$ & $-0.10 \pm 0.21$ \\
\hline $\mathrm{L}^{-\mathrm{mPEG}_{114}-\mathrm{DSPE}}$ & $177 \pm 2.40$ & $0.16 \pm 0.01$ & $-0.78 \pm 0.48$ \\
\hline L-mPEG $114-$ Chol & $164 \pm 1.15$ & $0.12 \pm 0.01$ & $-2.18 \pm 0.67$ \\
\hline L-mPEG $114-$ Chln & $167 \pm 11.8$ & $0.15 \pm 0.02$ & $-2.23 \pm 0.83$ \\
\hline $\mathrm{B}-\left(\mathrm{mPEG}_{114}\right)_{2}-\mathrm{DSPE}$ & $180 \pm 3.38$ & $0.09 \pm 0.01$ & $-1.02 \pm 0.43$ \\
\hline
\end{tabular}

${ }^{a}$ All the analysis were performed at a concentration of $1 \mathrm{mg} / \mathrm{mL}$ in $10 \mathrm{mM}$ phosphate, $140 \mathrm{mM} \mathrm{NaCl}, \mathrm{pH}$ 7.4. Liposomes were labelled with 0.5 mol\% Rho-DHPE.

The TEM analyses reported in Figure 3C and Figure S1a (Supplementary Information) confirmed the size and the polydispersity of the PEGylated formulations determined by DLS. TEM images of the naked vesicles showed a few aggregates, which explain the high PDI observed by DLS (Figure S2 in Supplementary Information). The stabilizing effect provided by the PEG coating can minimize aggregation as required for pharmaceutical liposomes. The zetapotential was found to be slightly negative for all liposomes except for cationic liposomes.
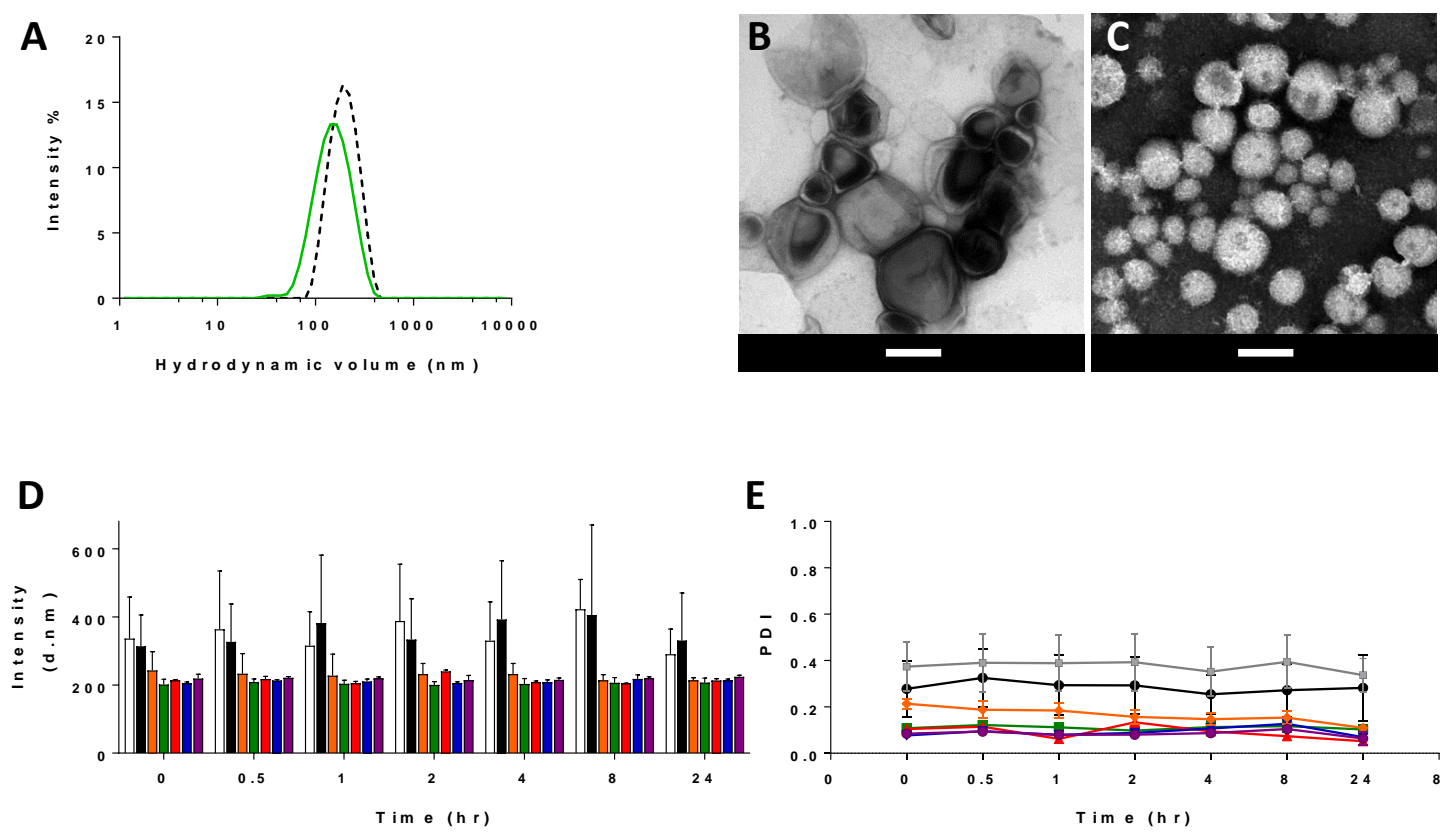
Figure 3. Representative DLS size analysis profiles (A) of naked (- -) and L-mPEG $114-\mathrm{DSPE}$ coated liposomes (-). TEM images of a $1 \mathrm{mg} / \mathrm{mL}$ suspension in PBS, $\mathrm{pH} 7.4$, of (B) naked and (C) L-mPEG $114-D S P E$ coated liposomes. Scale bar is $200 \mathrm{~nm}$. Mean size expressed in Intensity (D) and polydispersity index (E) of naked liposomes ( $\square$ ), cationic liposomes ( $(\mathbf{)})$ and liposomes

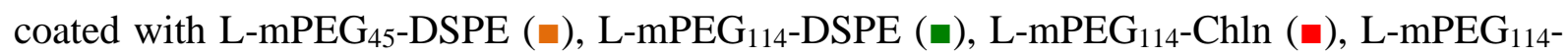
Chol ( $\square$ ) or B-[mPEG $\left.{ }_{114}\right]_{2}-\mathrm{DSPE}(\boldsymbol{m})$ at the concentration of $1 \mathrm{mg} / \mathrm{mL}$ in PBS added of $2.5 \%$ v/v FBS. Data were recorded by DLS analysis at 0, 0.5, 1, 2, 4, 8 and 24 hours.

Since protein adsorption on liposome surface (opsonization) can significantly affect the vesicle size and surface properties that dictate the the liposome stability, DLS studies were performed in the presence of FBS.

The DLS results summarized in Figure 3D and 3E show that the size of all PEGylated liposomes and PDI remained constant throughout the time in the presence of serum proteins indicating that the coating agents possess similar shielding performance under these experimental conditions. On the contrary, naked and cationic liposomes showed a remarkable size and PDI increase soon after liposome contact with serum, ${ }^{57-59}$ which can be attributed to either serum protein mediated liposome/liposome bridging or by protein layer adsorption on the liposome surface.

\subsection{In vitro studies}

Liposome toxicity was evaluated in vitro by hemolytic test, cell viability assays and TNF- $\alpha$ release. Indeed, a few studies indicate that naked and PEGylated liposomes possess haemolytic activity depending on composition, concentration, and incubation time. ${ }^{60,61}$ Also, literature reports that naked and PEGylated liposomes may decrease cell viability. ${ }^{62,63}$ Furthermore, at best of our knowledge, no information was found about the in vitro toxicity of branched[mPEG $]_{2}$-DSPE coated liposomes. Thus, aimed at investigating the effect of different PEGylating agents on liposome toxicity, a comparative cytotoxicity and haemolytic studies was carried out.

In hemolytic studies, SDS and dextran were used as positive and negative control, respectively. Figure 4A shows that cationic liposomes yielded remarkable dose-dependent hemolysis as a consequence of the charge mediated interaction with the negatively charged membranes of erythrocytes. ${ }^{64}$ Naked vesicles showed a limited hemolytic activity $(\sim 10 \%)$ even at high 
concentrations. All PEG-coated liposomes showed excellent biocompatibility at all the experimental concentrations. Nevertheless, the hemocompatibility seems to be affected by the anchoring moiety. Indeed, by comparing the results obtained with PEGylating agents of the

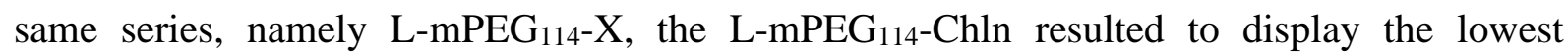

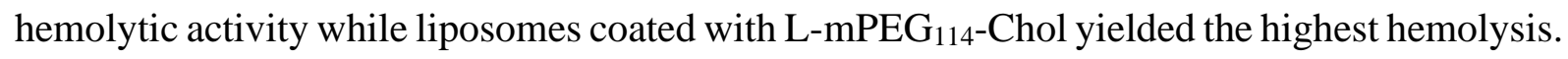
Possibly, L-mPEG $114-$ Chol has higher ability to exchange from the liposome bilayer to biological membranes resulting in their destabilization. By comparing the results obtained with PEGylating agents containing the same anchoring group, mPEG-DSPE series, it is possible to observe that the polymer size and shape does not affect the hemolytic properties of the coating liposomes. 
A

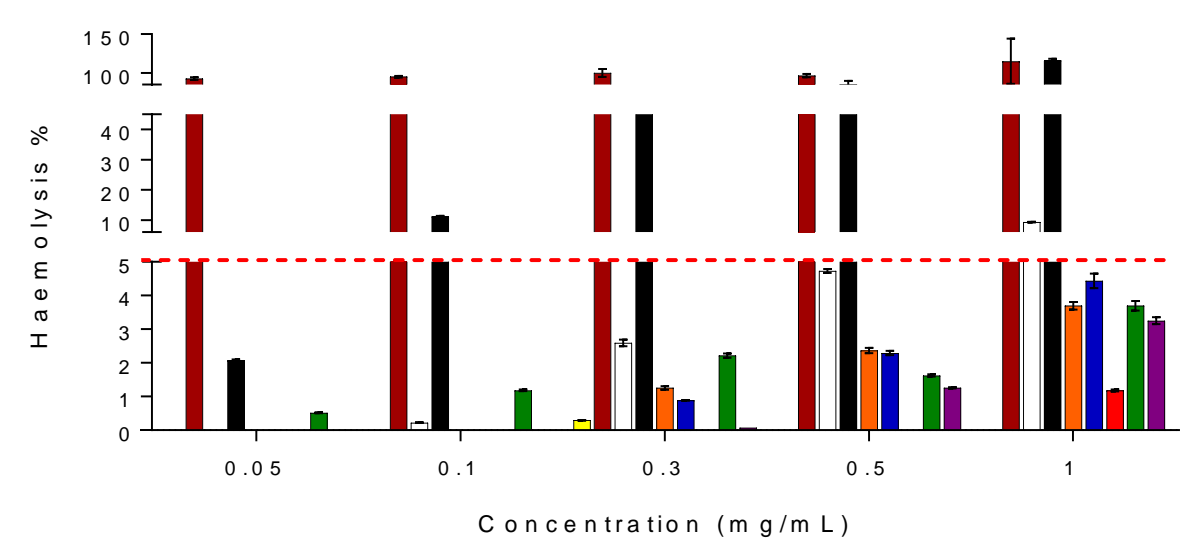

B

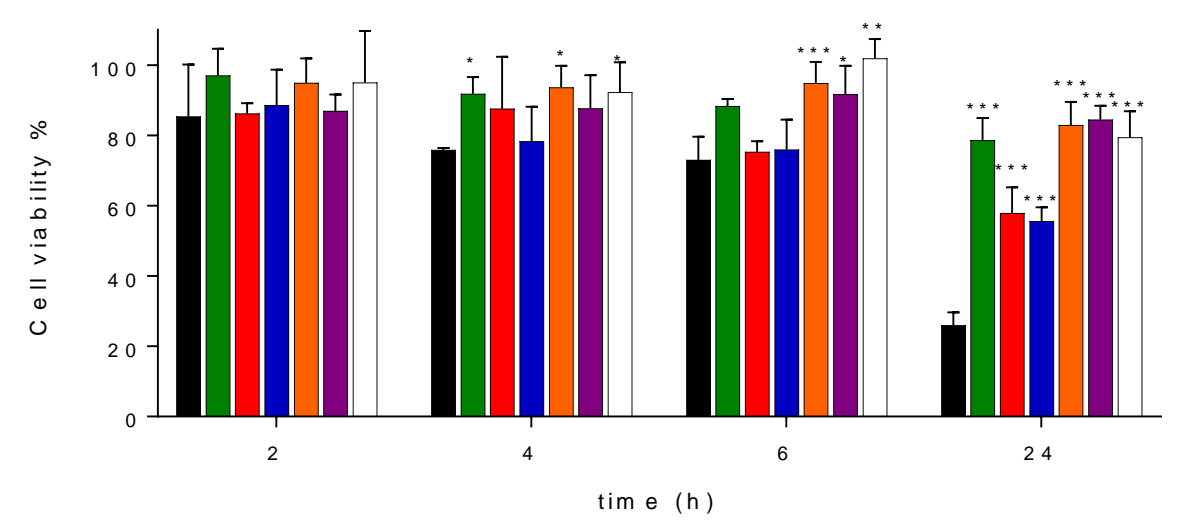

C

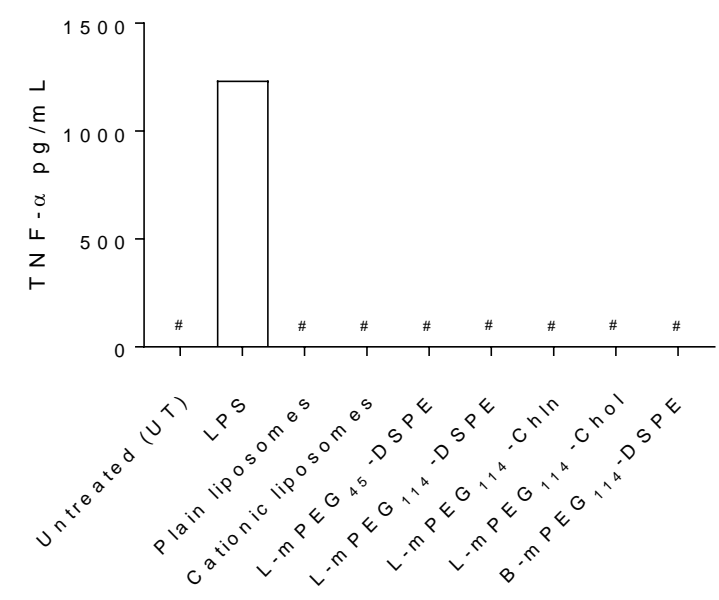

Figure 4. Hemolysis (A) and Hela cells viability (B) profiles, and TNF- $\alpha$ release from isolated PBMC (C) after incubation with naked liposomes ( $\square$ ), cationic liposomes ( $\mathbf{\square})$ and liposomes

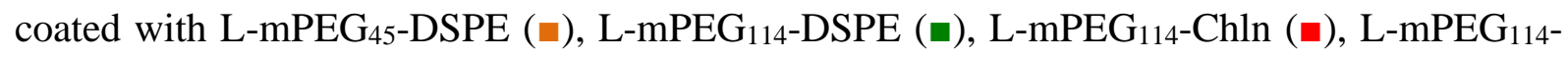

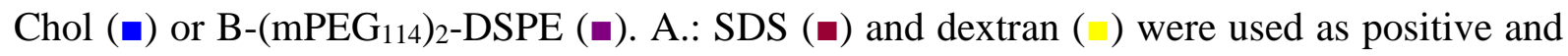
negative controls, respectively, in the hemolytic study. B.: Cell viability was assessed at 1 $\mathrm{mg} / \mathrm{mL}$ liposomes. C.: TNF- $\alpha$ release from isolated PBMC was assessed after 6 hours incubation with $1 \mathrm{mg} / \mathrm{mL}$ liposomes. Untreated cells and cells treated with LPS were used as negative and positive control, respectively. Detection limit: $15.6 \mathrm{pg} / \mathrm{mL}$. Values below the detection limit are marked with "\#”. 
Statistical significance: $n s \mathrm{p}>0.05,{ }^{*} \mathrm{p}<0.05,{ }^{*} \mathrm{p}<0.01, * * * \mathrm{p}<0.001$.

Figure 4B shows that after 24 hours incubation with Hela cells, cationic liposomes displayed a remarkable cytotoxicity with $74 \%$ decrease of cell viability. In this case also, the biocompatibility of the liposomes can be associated to the chemical nature of the anchoring group rather than to the polymer size and structure. Indeed, all the DSPE derivatives yield liposomes inducing negligible reduction of cell viability (no differences were observed with the

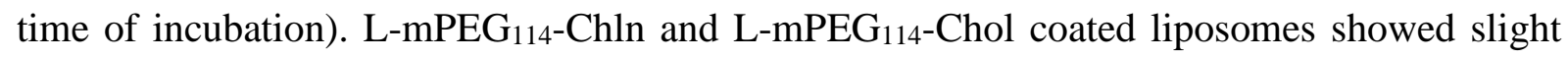
and comparable reduction of the cell viability in the first $6 \mathrm{~h}$ while at $24 \mathrm{~h}$ a decreased viability was observed. These results indicate that exchange and interaction of the mPEG-lipids with biological membranes is affected by their composition, which can explain the different behavior of these two materials in term of toxicity toward both erythrocytes and cancer cells. ${ }^{65}$

The safety of the liposomes was also evaluated by assessing the release of the pro-inflammatory cytokine TNF- $\alpha$ by monocytes, which is an indicator of mononuclear cell activation that may occur after exposure to pathogenic microorganisms and drug carriers. ${ }^{66,67}$ The assay was performed by incubating the liposomes with peripheral mononuclear blood cells (PBMC) using LPS as positive control. The results reported in Figure $4 \mathrm{C}$ show that none of the liposome formulation triggered TNF- $\alpha$ release demonstrating the safety of the nanocarriers towards the cells involved in inflammation.

The stability and stealth properties conferred to liposomes by PEG coating is expected to prevent their interaction with cells thus reducing off-target disposition of the carriers. The effect of PEG architecture and length and the lipid anchor structure on cell association of liposomes was evaluated by flow cytometry after incubating cells with liposomes in the presence of serum. Figure 5 shows that naked vesicles undergo a time dependent cell uptake. Up to $6 \mathrm{~h}$ of incubation a limited cell uptake was observed that remarkably increased over 24 hours. This behavior may be ascribed to the serum protein adsorption on the uncoated liposome surface, which promotes unspecific protein mediated cell adhesion. Cationic liposomes showed even worst behavior than naked liposomes. Cationic liposomes immediately associate extensively with cells and then the association slightly increases throughout the incubation time. The high cell association of the cationic liposomes is in agreement with the surface charge interaction due to the positive charge of the vesicle and the negative charge of the cells..$^{57,68}$ 
A
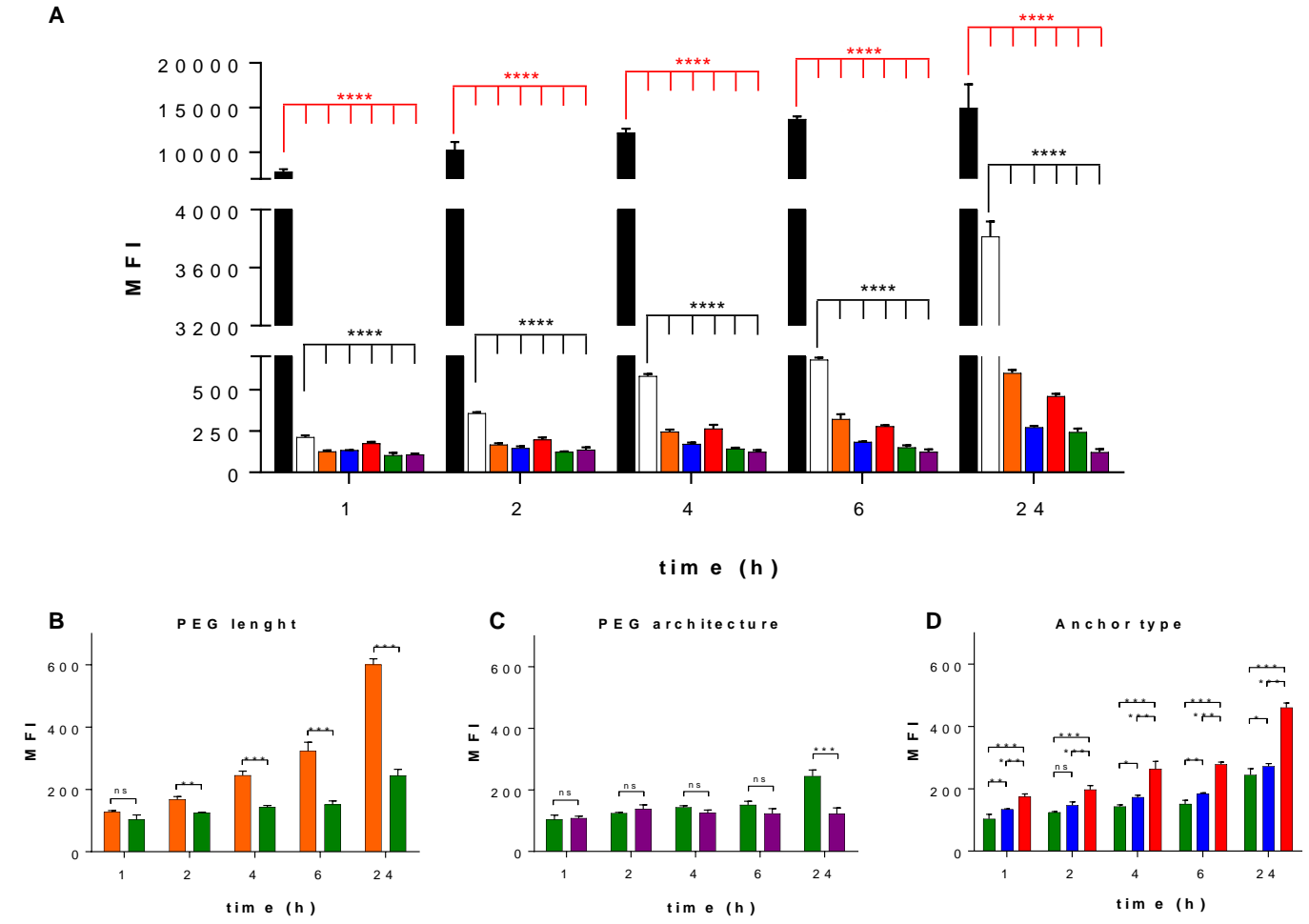

Figure 5. (A) Flow cytometric analysis of Hela cells incubated with $1 \mathrm{mg} / \mathrm{mL}$ suspension of

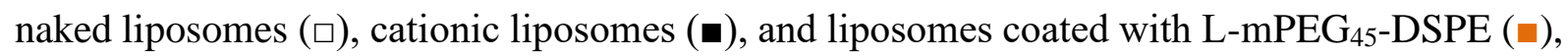

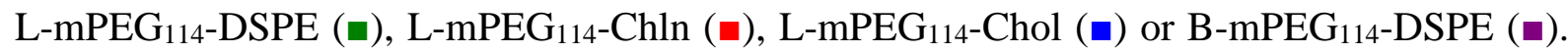
Statistical significance of MFI of cells incubated with naked and PEGylated liposomes was calculated $v s$ the MFI of cells incubated with cationic liposomes (red bars); statistical significance of MFI of cells incubated with PEGylated liposomes was calculated $v s$ the MFI of cells incubated with naked liposomes (black bars): ${ }^{* * * *} \mathrm{p}<0.0001$.

Expanded details of the liposome association profiles with Hela cells obtained by comparing: (B) PEG length; (C) PEG architecture; anchor type (D). Statistical significance: ns p>0.05, $* \mathrm{p}<0.05, * * \mathrm{p}<0.01, * * * \mathrm{p}<0.001$.

As expected, all PEGylated liposomes showed lower cell association than naked and cationic liposomes. However, Figure 5B shows that in the same series of coating agents, mPEG-DSPE, shorter PEG (L-mPEG45-DSPE) yielded higher cell association than longer PEG (L-mPEG $114-$ DSPE), which is probably due to a slight lower shielding capacity provided by the shorter PEG chain. ${ }^{69}$ Liposomes coated with L-mPEG $114-\mathrm{DSPE}$ showed a $\sim 16$-fold decrease of association with cells with respect to the naked liposomes . 
Figure 5C shows that liposomes coated with branched PEG [B-(mPEG $\left.\left.{ }_{114}\right)_{2}-\mathrm{DSPE}\right]$, which have been obtained by anchoring half moles of the PEGylating agent on the liposomes surface than in the case of the linear derivatives in order to achieve the same chain density, were found to associate to cells at lower extent with respect to liposomes coated with linear polymers. The association of these liposomes to cells was $\sim 31$ times lower than naked liposomes. This result is in good agreement with the literature data demonstrating that branched polymers shield more efficiently the liposome surface thus preventing the protein adsorption responsible for vesicle association to cells. ${ }^{70}$

Figure 5D shows that the anchoring group also plays a role in the liposome association to cells. A comparative evaluation of the results obtained with liposomes coated with mPEG functionalized with the different anchoring groups (DSPE, Chol and Chln) showed that L$\mathrm{mPEG}_{14-\mathrm{DSPE}}$ and $\mathrm{L}_{-\mathrm{mPEG}} \mathrm{P}_{14-}$-Chol yield vesicles with the lowest cell association properties while L-mPEG ${ }_{114}-\mathrm{Chln}$ yields liposomes with higher cell association. These results indicate that DSPE and cholesterol promote more stable association of the PEGylating derivative with the liposome bilayer than cholane suggesting that the alkyl chains (stearic acid moieties of DSPE and dimethyl-6-methylheptan2-yl of Chol) favor the anchoring stability. On the contrary, the conjugation of mPEG to the alkyl chain of cholane, which in the case of cholesterol is located in the core of the lipid bilayer ${ }^{71}$ may constrain an unfavorable orientation of the cholane moiety within the lipid bilayer with consequent weak association to the bilayer. Overall L$\mathrm{mPEG}_{114}$-Chol coated liposomes undergo 14-times lower association to cells compared to naked liposomes.

The vesicle disposition within cells was further investigated by confocal microscopy after 6 hours incubation with Rho-liposomes. In Figure 6, the cationic liposomes showed high fluorescence associated to cells compared to naked liposomes. The zeta stack analysis confirmed that when liposomes associate to cells they dispose mainly in the cytosol as a result of cell uptake (Figure S3 in Supplementary Information). All PEGylated liposomes did not show cell fluorescence, which agrees with the results reported above. 

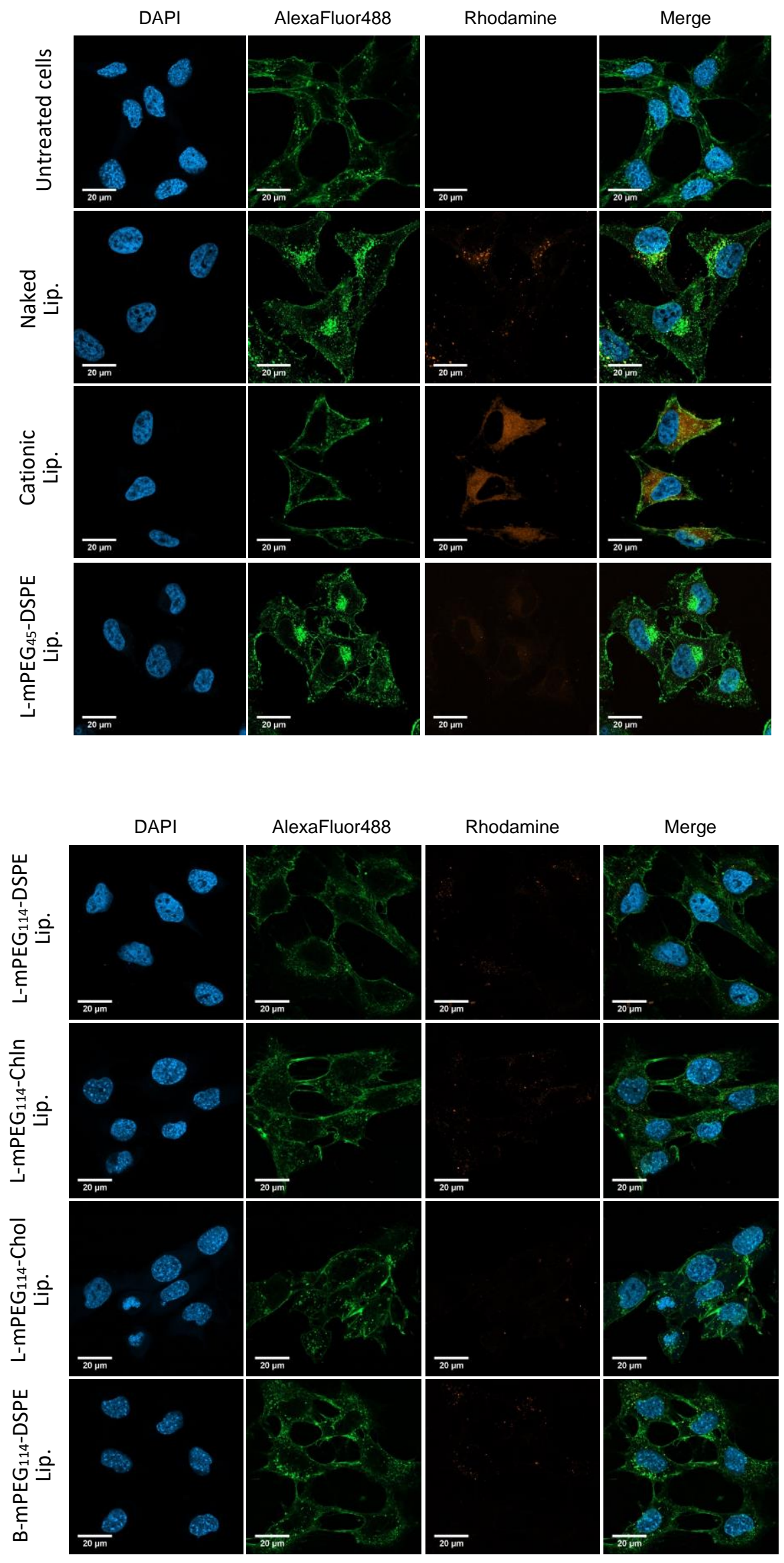
Figure 6. Confocal images of HeLa cells incubated with $1 \mathrm{mg} / \mathrm{mL}$ suspension of naked liposomes, cationic liposomes and liposomes coated with L-mPEG 45 -DSPE, L-mPEG $114-\mathrm{DSPE}$, L-mPEG ${ }_{114}-\mathrm{Chln}, \mathrm{L}_{-} \mathrm{mPEG}_{114}-\mathrm{Chol}$ or B-[mPEG$\left.{ }_{114}\right]_{2}$-DSPE. Nucleus was labelled with DAPI (blue), cell membrane with (WGA)-AlexaFluor® 488 (green) and liposomes with RhodamineDHPE (red).

\subsection{Pharmacokinetic study}

Extended circulation time in the bloodstream of drug nanocarriers is a requisite to promote their passive disposition into solid tumors or into inflamed tissues according to EPR effect. ${ }^{72}$ Furthermore, this feature, in the absence of drug leakage from the nanocarrier, reduces the biodistribution of the payload to off-target sites and rapid clearance due to RES cell uptake.

PK studies were performed by labelling liposomes with rhodamine-DHPE. A preliminary validation study showed that $>96 \%$ of rhodamine-DHPE used for liposome labelling was recovered after $24 \mathrm{~h}$ incubation with buffer, plasma or whole blood (Figure S4 and S5 in Supplementary Information) indicating that rhodamine-DHPE does not associates with erythrocytes or serum proteins.

The PK profiles of liposomes intravenously administered to BALB/c mice reported in Figure 7 were found to fit a two-compartment model ${ }^{73}$ The PK parameters are reported in Table II. In general, all PEGylated liposomes showed longer distribution phase $\left(t_{1 / 2} \alpha\right)$, shorter elimination phase $\left(t_{1 / 2} \beta\right)$, higher clearance $(C L)$ and smaller overall systemic bioavailability (AUC), with respect to naked liposomes. However, a few differences observed in the PK data indicate that the structure of the PEGylating agents plays a role in the in vivo liposome fate. The comparative results obtained with liposomes coated with mPEG-DSPE derivatives, showed that the polymer length and polymer architecture (linear or branched) on the vesicle surface have remarkable effect on the liposome PK profiles. In particular, the results obtained with liposomes coated with L-mPEG 45 -DSPE showed they have slower elimination phase $\left(\mathrm{t}_{1 / 2} \beta\right)$ and lower clearance than $\mathrm{L}_{-\mathrm{mPEG}} \mathrm{PL}_{14}$-DSPE coated liposomes, resulting in longer permanence in the bloodstream. This result is rather unexpected because the two liposome formulations possess similar hydrodynamic size and in vitro stability. Therefore, according to the results obtained with L-mPEG $45-\mathrm{DSPE}$ and L-mPEG ${ }_{114}-\mathrm{DSPE}$, it seems reasonable to think that longer is the PEG chain easier is the detachment under the shear forces in the circulation, which can reflect on partial loss of stealth properties. The liposomes coated with B-(mPEG 114$)_{2}$-DSPE displayed even lower permanence in the circulation than L-mPEG 114 -DSPE coated liposomes, 
suggesting that also the polymer architecture plays a role in the PK profile of the liposomes. However, according to previous hypothesis, it is reasonable to derive that the lower performance of the liposomes coated with $\mathrm{B}-\left(\mathrm{mPEG}_{114}\right)_{2}-\mathrm{DSPE}$ is due to the high polymer mass attached to a single anchoring group, which in the case of B-(mPEG $\left.{ }_{114}\right)_{2}-\mathrm{DSPE}$ is double of LmPEG$_{14-D S P E}$. This is in agreement with comparative studies of linear and branched polyether-lipids coated liposomes in the literature, where the branched polyether-lipids displayed faster clearance with respect to the linear counterparts. ${ }^{74}$
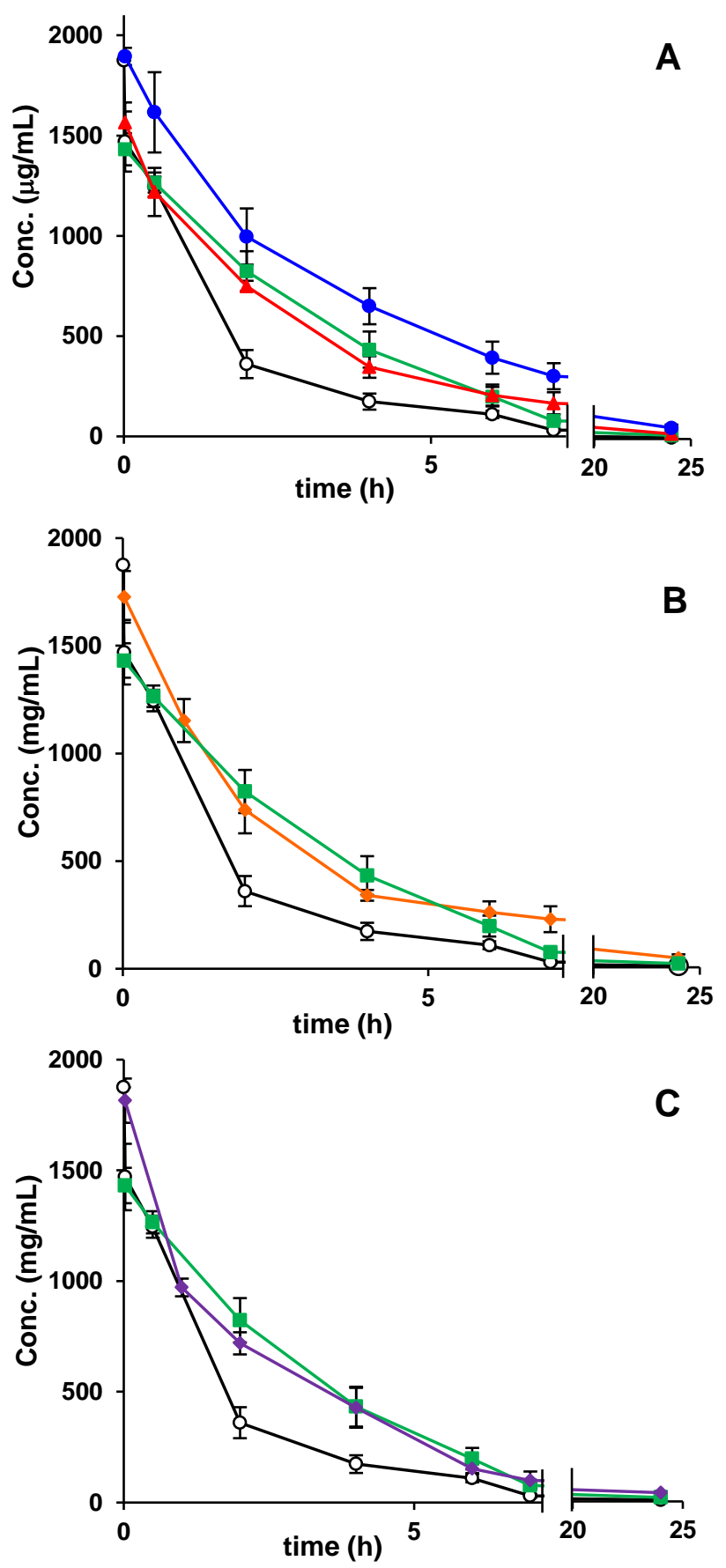
Figure 7. Blood concentration profile of Rho-liposomes detected after intravenous injection in mice. A. Profile of Rho-liposomes decorated with PEG agents with different lipid anchor. B. Profile of Rho-liposomes decorated with Linear PEG with different length. C. Profile of Rholiposomes decorated with PEGylating agents with different architecture. Liposomes coated with

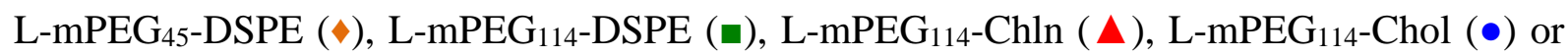
B-(mPEG $\left.{ }_{144}\right)_{2}$-DSPE $(\diamond)$ and naked liposomes (०).

Table II. Pharmacokinetic parameters obtained by applying the compartmental model after intravenous injection of Rho-liposomes in mice. Statistical significance analysis is reported in Table S3 in the Supplementary Information.

\begin{tabular}{|c|c|c|c|c|c|c|c|c|}
\hline Formulations & $\begin{array}{c}\mathrm{C}_{0} \\
(\mu \mathrm{g} / \mathrm{mL})\end{array}$ & $\begin{array}{c}\alpha \\
\left(\mathbf{t}^{-1}\right)\end{array}$ & $\begin{array}{c}\mathbf{t}_{1 / 2} \boldsymbol{\alpha} \\
(\mathbf{h})\end{array}$ & $\begin{array}{c}\beta \\
\left(\mathbf{t}^{-1}\right)\end{array}$ & $\begin{array}{c}\mathbf{t}_{1 / 2} \boldsymbol{\beta} \\
(\mathbf{h})\end{array}$ & $\begin{array}{c}\mathbf{C L} \\
(\mathrm{mL} / \mathrm{h})\end{array}$ & $\begin{array}{c}\mathrm{AUC}_{0-\infty} \\
\left(\mu \mathrm{g} / \mathrm{mL} \mathbf{L}^{*} \mathrm{~h}\right)\end{array}$ & $\begin{array}{c}\text { Normalized } \\
\text { AUC } \mathbf{C}_{-\infty} \\
\left(\mu \mathrm{g} / \mathbf{m L}^{*} \mathbf{h}\right)\end{array}$ \\
\hline Naked & 1875 & 857.14 & 0.001 & 0.4632 & 1.50 & 0.707 & 2652.8 & 2652.8 \\
\hline $\begin{array}{l}\text { L-mPEG }{ }_{45}- \\
\text { DSPE }\end{array}$ & 1755 & 0.54 & 1.280 & 0.0691 & 10.04 & 0.274 & 6846.2 & 7314.3 \\
\hline $\begin{array}{c}\text { L-mPEG }{ }_{114}- \\
\text { DSPE }\end{array}$ & 1470 & 0.32 & 2.175 & 0.3187 & 2.17 & 0.406 & 4612.6 & 5882.6 \\
\hline $\begin{array}{c}\text { L-mPEG }_{114}- \\
\text { Chol }\end{array}$ & 1901 & 0.41 & 1.702 & 0.0949 & 7.30 & 0.221 & 8498.0 & 8381.7 \\
\hline $\begin{array}{c}\text { L-mPEG }{ }_{114}- \\
\text { Chln }\end{array}$ & 1550 & 0.45 & 1.533 & 0.0881 & 7.87 & 0.358 & 5242.6 & 6341.8 \\
\hline $\begin{array}{c}\left.\text { B-[mPEG }{ }_{114}\right]_{2}- \\
\text { DSPE }\end{array}$ & 1968 & 13.46 & 0.052 & 0.3211 & 2.16 & 0.439 & 4266.5 & 4064.8 \\
\hline
\end{tabular}

The results obtained with $\mathrm{L}-\mathrm{mPEG}_{114}-\mathrm{Chol}_{\text {, }} \mathrm{L}-\mathrm{mPEG}_{114}-\mathrm{Chln}$ and $\mathrm{L}-\mathrm{mPEG}_{114}-\mathrm{DSPE}$ coated liposomes show that the anchoring group has a remarkable relevance in dictating the PK profile

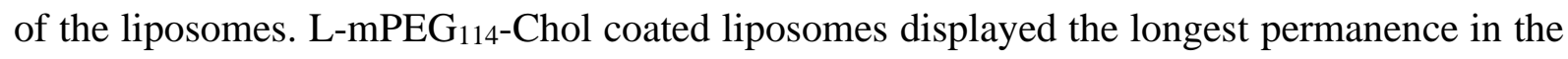
circulation, which is in agreement with the in vitro evidences demonstrating that $\mathrm{L}-\mathrm{mPEG}_{114}-$ Chol bestow liposomes with efficient stealth properties. The shorter residence time in the blood of the L-mPEG ${ }_{114}-$ Chln coated liposomes can result from the faster polymer detachment from the lipid bilayer upon administration in the blood stream as consequence of the lower stability of the liposome bilayer (as explained below by the in silico simulations) upon decoration with the $\mathrm{L}_{-\mathrm{PPEG}} \mathrm{H}_{14}-\mathrm{Chln}$, which results in faster clearance of these liposomes. 
It should be noted that PEGylated liposomes may undergo clearance by ABC (accelerated blood clearance), which is related to the humoral immunoresponse after multiple PEGylated liposome administration. All liposomes investigated in this study have been coated with same amount of PEGylating agents in order to minimize the effect of PEG content on the liposome surface on ABC. Nevertheless, different ABC could result from the different conformation of the PEG chains, which depends on the mol wt and structure, and the different stability of the decorating agents on the liposome surface.

\subsection{Computational studies}

Equilibration of simulated systems and properties calculated. For all seven systems simulated (see Methods section) the time taken to reach equilibrium was determined by measuring, as a function of simulation time, the number of contacts between $\mathrm{Na}^{+}$ions and the oxygen atoms of PEG and the lipid headgroups, in addition to the area per lipid. Salt $(\mathrm{NaCl})$ dissolved in water was included in the simulations to simulate more accurately the liposome surface in the physiological environment, i.e. the bloodstream. As an output of this calculation, we obtained average values of these properties (lipid area and $\mathrm{Na}^{+}$binding), with standard deviation, from the results in the latter $350 \mathrm{~ns}$ of the simulations for the six new simulated systems (Table S4 in the Supplementary Information). The "bound ions" refer to the total number of bound ions in each system per leaflet. As described in Methods, we calculated the mass density profile and electrostatic potential along the membrane normal and the deuterium order parameter along the length of the lipid chains. Snapshots (Figure 8A) of the final configuration of the trajectory for the seven systems that have been calculated.

Mass density profiles for all single components of the lipid bilayer (ions, phosphate headgroups, PEG, lipids, solvent, etc...) have been determined (Figure S6 In Supplementary Information). The results for two specific components, namely the PEG corona and the $\mathrm{Na}^{+}$ions, are key to understand the behavior of the systems (Figure 8B).

Mass density profile of PEG: length of PEG and branching. The mass density profile results for PEG for the systems with $\mathrm{mPEG}_{45}, \mathrm{mPEG}_{114}$ and $\left[\mathrm{mPEG}_{114}\right]_{2}$ (see Figure $8 \mathrm{~B}$ ) show that, as expected, the PEG coating layer is considerably thicker for the systems including linear $\mathrm{mPEG}_{114}$ and branched $\left(\mathrm{mPEG}_{114}\right)_{2}$ than for the systems obtained with linear $\mathrm{mPEG}_{45}$, despite the similar polymer density on the vesicle surface; the thickness of the PEG layer on the lipid membrane, for the case of linear mPEG-DSPE, roughly linearly correlates with the PEG chain length (namely 45 and 114 ethylene glycol monomers). The relative thickness of the polymer 
corona as a function of polymer length thus follows the expected results for a polymeric coating in the brush regime, in agreement with Alexander - de Gennes theory. ${ }^{15,} 75$ According to the literature, the transition between the mushroom and brush conformations is around $5 \mathrm{~mol} \%$ grafting density, which was used in both experimental studies reported above and simulations to generate linear $\mathrm{mPEG}_{45}$ and $\mathrm{mPEG}_{114}$ coated liposomes. ${ }^{49}$ Thus, this behavior is in agreement with the literature reports and indicates that the arrangement of the PEG layer under these conditions is in the brush conformation in our simulation.

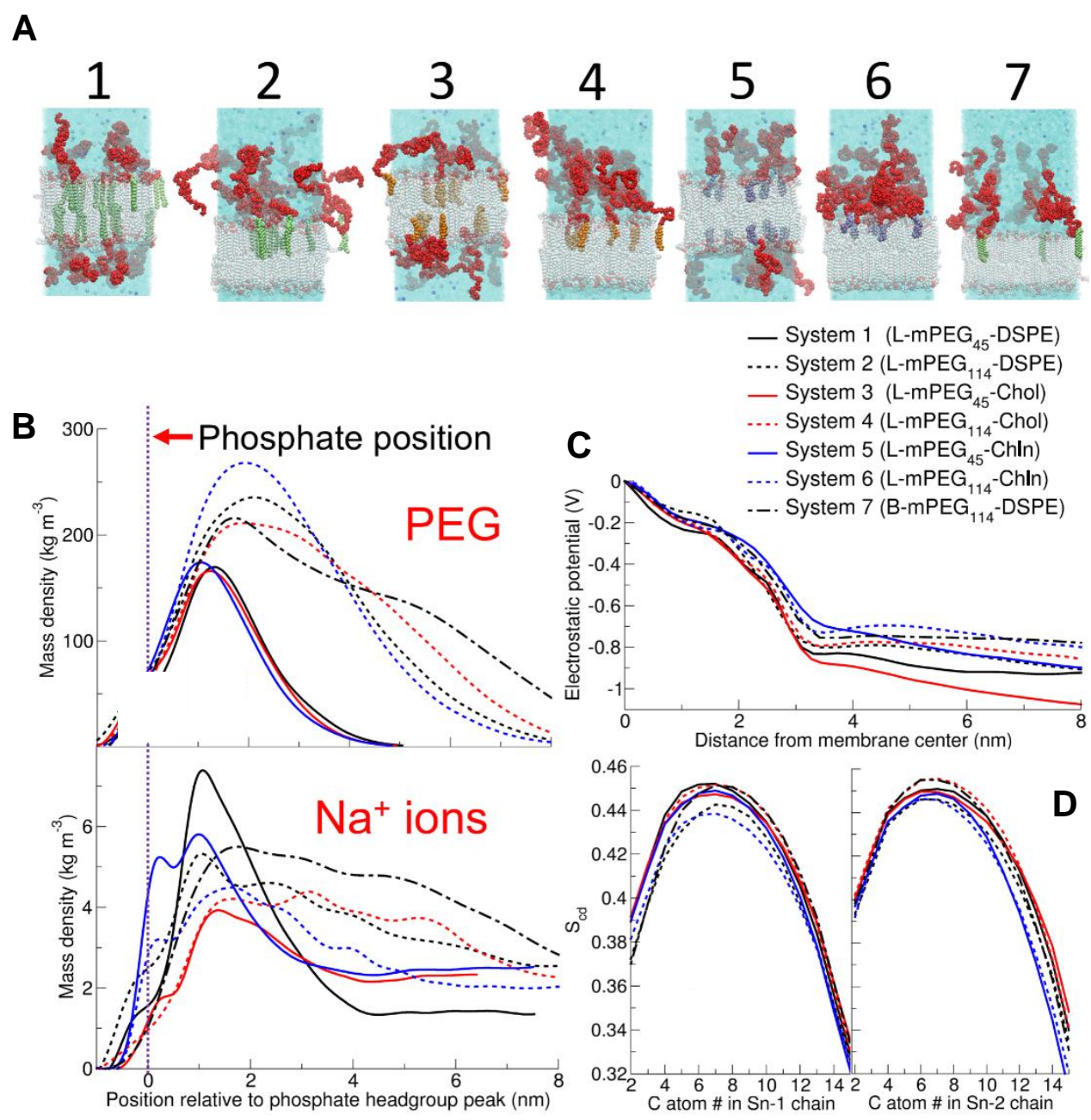

Figure 8. A. Snapshots of the final configuration of the simulated trajectories of the seven systems. In all systems obtained with $\mathrm{PEG}_{114}$ coating only one leaf of the membrane was PEGylated due to the system size needed. B. Mass density profile results. C. Charge density profile results. D. Deuterium order parameter. 
Interestingly, the mass density profile of the PEG layer for the branched PEGylating agent is even thicker than that for linear $\mathrm{mPEG}_{114}$ and the result for the mass density profile (Figure 8B) shows a double peak structure. This is probably the result of additional PEG aggregation which is facilitated by the closer proximity of the PEG chains near the membrane headgroups due to the branched structure of the molecule. Also, the branched PEG exhibits greater density further from the membrane than the case of linear $\mathrm{mPEG}_{114}$. This is in agreement with results reported in the literature that show an increased protective efficiency of branched PEG derivatives on the surface of liposomes. ${ }^{76}$

Mass density profile of PEG: Molecule to which PEG is functionalized. The mass density profile results for PEG in the systems with PEG functionalized with DSPC (transformed to DSPE), cholesterol and cholane (see Figure 8B) show that the PEG layer on the membrane surface where mPEG is functionalized with the cholane is disposed closer to the membrane headgroups than for the membranes with mPEG functionalized with the phospholipid or cholesterol. It can be hypothesized that this is the result of a looser lipid membrane structure than in the case of L-mPEG $114-\mathrm{DSPE}$ and $\mathrm{L}_{-} \mathrm{mPEG}_{114}-\mathrm{Chol}_{\text {, as }}$ the interaction between the PEG polymer and the membrane surface is increased. This conclusion has been confirmed by the charge profile and deuterium order parameter results reported below.

Mass density profile of $\mathrm{Na}^{+}$ions. As $\mathrm{Na}^{+}$ions are known to bind to the PEG oxygens ${ }^{28}$ in addition to the phosphate groups in the lipids, their mass density profile roughly matches that of the PEG polymer, with an additional secondary peak at the position of the phosphate headgroups (Figure 8B). Strikingly, this secondary peak of mass density raised by $\mathrm{Na}^{+}$ associated to the phosphate groups is much stronger in the membrane that includes the $\mathrm{mPEG}_{114}-\mathrm{Chln}$ indicating a better access to the lipid headgroups, thus a looser lipid membrane structure (see Table S4 in Supplementary Information) with a significantly larger number of $\mathrm{Na}^{+}$ions bound to the phosphate headgroups.

Electrostatic potential and deuterium order parameter results. The electrostatic potential results shown in Figure $8 \mathrm{C}$ indicate that the potential change from the inner part to the surface of the membrane is considerably lower in membranes coated with $\mathrm{mPEG}_{114}-\mathrm{Chln}$ than with other PEGylating agents. This confirms a looser, less ordered, membrane structure. Results for the deuterium order parameter along the lipid chains for all seven systems are shown in Figure 8D, for both the Sn-1 and Sn-2 acyl chains of the DSPE. Further down towards the ends of the lipid tails a consistent decrease of deuterium order parameter is observed in the presence of $\mathrm{L}-\mathrm{mPEG}_{114}-\mathrm{Chln}$ with respect to the membrane including the other PEGylating agents. This, once again, indicates a decreased lipid order. 
Altogether, the computational MD modelling results point out that the membrane coated with L-mPEG ${ }_{114}$-Chln present a looser and less ordered lipid membrane structure. This indicates lower structural stability; this is in agreement with the evidence from the in-vitro and in-vivo results showing that the liposomes coated with mPEG-Chln derivatives have less stealth properties, i.e. protective, than all other PEGylating agents studied in this work.

Orientation of cholane vs. cholesterol in the membrane. For what concern the polycyclic anchoring groups, cholane has similar structure of cholesterol (Figure 2). However, the mPEGChln derivatives can associate to the lipid membrane in two possible conformations. In the first case, the cholane moiety in the liposome bilayer has inverted orientation with respect to cholesterol of mPEG-Chol derivatives, which implies that the cholane carbonyl group where mPEG is attached is directed outwards from the membrane. In the second case the cholane moiety within the bilayer is oriented similarly to the cholesterol moiety of the mPEG-Chol derivatives with the carbonyl group embedded in the lipid bilayer. In such a case the PEG chain is forced to twist outwards from the membrane, running alongside the Cholane molecule. To test these hypotheses, we ran $200 \mathrm{~ns}$ long simulations with 5 replicas of the L-mPEG ${ }_{114}-\mathrm{Chln}$ in both possible orientations. As can be measured by the angle between the longitudinal axis of the cholane molecules and the membrane normal, we observed, in all five replica systems, that the cholane moiety orients in the inverted orientation with respect the cholesterol moiety (data not shown); this is in agreement with our hypothesis regarding the effect of PEGylation on the membrane ordering properties of cholane. We thus conclude that the L-mPEG $114-\mathrm{Chln}$ association with lipid membrane generate less stable conditions within the membrane than L$\mathrm{mPEG}_{114-\mathrm{Chol}}$. Indeed, the polycyclic molecule is forced to invert orientation with respect to the one that would embed it into the membrane as firmly as the cholesterol molecule. Although cholane (without carboxyl group) has been shown to sit within the membrane core without a preferred orientation as a result of its lack of polar groups (Figure SI7), in the case of mPEGChln the linker region, where PEG is conjugated to the polycyclic lipid, includes a polar carbonyl group similarly to the mPEG-Chol derivatives. However, although the structure of cholane is similar to that of cholesterol, when cholane is lined up with cholesterol the site of the linker region is at the opposite end of the molecule with respect to the case for cholesterol. Thus, the resulting orientation of Chln in the mPEG-Chln derivatives associated to the lipid bilayer is, effectively, the reverse of that of cholesterol, and unable to play the same role as cholesterol plays in ordering the membrane, thus explaining the more weakly ordered bilayers with respect to the bilayers coated with $\mathrm{mPEG-Chol} \mathrm{derivatives.}$ 


\section{CONCLUSIONS}

The overall in vitro and the PK results reported in this work indicate that the different performances of PEG-lipids in providing stealth features to liposomes in vitro may not closely reflect in the circulation time enhancement in the blood stream in vivo. Taken together, the results suggest that both in vitro stability and long circulation of liposomes require a balance of the polymer molecular weight/architecture and the association strength of the lipidic anchor to vesicle bilayer. The in vivo results suggest that high size PEG chains (long linear or branched PEGs) of PEGylating agents are less efficient in improving the stealth features of liposomes with respect to low size PEG chains in vivo, likely because of lower resistance to shear stress arising during blood circulation. On the other hand, lipid anchors of the PEGylating agents that generate more ordered lipid bilayer structures remain associated to liposomes more firmly with beneficial effect on the stealth features. It is worth to note that phosphate groups, namely PEGphospholipids, and lipids that enhance the fluidity of liposome bilayer can increase the complement activation. ${ }^{11,77}$ Thus, we can speculate that neutral PEGylating agents that increase the order of the lipid bilayer, namely L-mPEG-Chol, are expected to reduce the liposome induced complement activation.

The evidences obtained in this study also highlight the need for suitable in vitro models to better recapitulate the environmental complexity found in vivo (i.e. blood), which ultimately can foster the replacement of animal experimentation for the development of stealth drug delivery systems. Additionally, computational modelling has been shown to have the capability to provide complementary mechanistic insight. However, it should be noted that since the stability of the coating on particle surface is affected by a variety of parameters that dictate the interaction of coating agents and particle surface, an accurate choice of PEGylating agents should include not only the chemical nature, composition and structure of the coating agent, but also the physicochemical nature of the particles that should be coated.

Despite PEG is considered the gold-standard for liposome coating, a variety of alternative polymers have been so far investigated for this application, including vinyl based polymers, poly(2-oxazoline)-polyglutamic acid (PGA), poly(hydroxyethyl-l-asparagine) (PHEA) and poly(hydroxyethyl-1-glutamine) (PHEG), zwitterionic polymers etc. ${ }^{40}$ In a few cases these polymers have been found to enhance the biopharmaceutical and pharmacokinetic properties of liposomes compared to PEGylation. For example, poly-(amino acid) derivatives were found to yield liposomes with lower ABC than PEGylated liposomes. ${ }^{78}$ However, whether these novel polymers can replace PEG and overcome its limitations it is still a matter of investigation. 


\section{SUPPLEMENTARY INFORMATION}

Supplementary information includes: additional equipment description; molecular force fields, simulation parameters and initialization procedure for computational molecular modelling; additional liposome formulation characterization, validation of liposome quantification procedures; in vitro additional confocal imaging; additional computational results.

\section{ACKNOWLEDGEMENTS}

All computational work was carried out using the resources of the CSC - IT Center for Science Ltd.

\section{REFERENCES}

1. Kudgus, R. A.; Walden, C. A.; McGovern, R. M.; Reid, J. M.; Robertson, J. D.; Mukherjee, P. Tuning pharmacokinetics and biodistribution of a targeted drug delivery system through incorporation of a passive targeting component. Scientific reports 2014, 4.

2. Mastrotto, F.; Salmaso, S.; Lee, Y. L.; Alexander, C.; Caliceti, P.; Mantovani, G. pHresponsive poly(4-hydroxybenzoyl methacrylates) - design and engineering of intelligent drug delivery nanovectors. Polymer Chemistry 2013, 4, (16), 4375-4385.

3. Mastrotto, F.; Salmaso, S.; Alexander, C.; Mantovani, G.; Caliceti, P. Novel pHresponsive nanovectors for controlled release of ionisable drugs. Journal of Materials Chemistry B 2013, 1, (39), 5335-5346.

4. Sercombe, L.; Veerati, T.; Moheimani, F.; Wu, S. Y.; Sood, A. K.; Hua, S. Advances and challenges of liposome assisted drug delivery. Frontiers in pharmacology 2015, 6.

5. Bulbake, U.; Doppalapudi, S.; Kommineni, N.; Khan, W. Liposomal Formulations in Clinical Use: An Updated Review. Pharmaceutics 2017, 9, (2), 12.

6. Nag, O. K.; Yadav, V. R.; Hedrick, A.; Awasthi, V. Post-modification of preformed liposomes with novel non-phospholipid poly (ethylene glycol)-conjugated hexadecylcarbamoylmethyl hexadecanoic acid for enhanced circulation persistence in vivo. International journal of pharmaceutics 2013, 446, (1-2), 119-129.

7. $\quad$ Gref, R.; Lück, M.; Quellec, P.; Marchand, M.; Dellacherie, E.; Harnisch, S.; Blunk, T.; Müller, R. 'Stealth' corona-core nanoparticles surface modified by polyethylene glycol (PEG): influences of the corona (PEG chain length and surface density) and of the core composition on phagocytic uptake and plasma protein adsorption. Colloids and Surfaces B: Biointerfaces 2000, 18, (3), 301-313.

8. Beroström, K.; Österberg, E.; Holmberg, K.; Hoffman, A. S.; Schuman, T. P.; Kozlowski, A.; Harris, J. M. Effects of branching and molecular weight of surface-bound poly (ethylene oxide) on protein rejection. Journal of Biomaterials Science, Polymer Edition 1995, $6,(2), 123-132$.

9. Abe, K.; Higashi, K.; Watabe, K.; Kobayashi, A.; Limwikrant, W.; Yamamoto, K.; Moribe, K. Effects of the PEG molecular weight of a PEG-lipid and cholesterol on PEG chain flexibility on liposome surfaces. Colloids and Surfaces A: Physicochemical and Engineering Aspects 2015, 474, 63-70. 
10. Webb, M. S.; Saxon, D.; Wong, F. M.; Lim, H. J.; Wang, Z.; Bally, M. B.; Choi, L. S.; Cullis, P. R.; Mayer, L. D. Comparison of different hydrophobic anchors conjugated to poly (ethylene glycol): effects on the pharmacokinetics of liposomal vincristine. Biochimica et Biophysica Acta (BBA)-Biomembranes 1998, 1372, (2), 272-282.

11. Moghimi, S. M.; Hamad, I.; Andresen, T. L.; Jørgensen, K.; Szebeni, J. Methylation of the phosphate oxygen moiety of phospholipid-methoxy (polyethylene glycol) conjugate prevents PEGylated liposome-mediated complement activation and anaphylatoxin production. The FASEB journal 2006, 20, (14), 2591-2593.

12. Uster, P. S. Liposomes as drug carriers: Recent trends and progress. Edited by Gregory Gregoriadis. John Wiley: Chichester, UK. 1988. xxvi+ 885 pp. $22 \times 16 \mathrm{~cm}$. ISBN 0-471-916544. Price not given. Journal of Pharmaceutical Sciences 1989, 78, (8), 693-693.

13. Papisov, M. I. Theoretical considerations of RES-avoiding liposomes: molecular mechanics and chemistry of liposome interactions. Advanced drug delivery reviews 1998, 32, (1), 119-138.

14. Magarkar, A.; Róg, T.; Bunker, A. Molecular dynamics simulation of PEGylated membranes with cholesterol: building toward the DOXIL formulation. J. Phys. Chem. B 2014, $118,(28), 15541-15549$.

15. Bunker, A.; Magarkar, A.; Viitala, T. Rational design of liposomal drug delivery systems, a review: Combined experimental and computational studies of lipid membranes, liposomes and their PEGylation. Biochimica et Biophysica Acta (BBA) - Biomembranes 2016, $1858,(10), 2334-2352$.

16. Pathak, P.; Dhawan, V.; Magarkar, A.; Danne, R.; Govindarajan, S.; Ghosh, S.; Steiniger, F.; Chaudhari, P.; Gopal, V.; Bunker, A.; Róg, T.; Fahr, A.; Nagarsenker, M. Design of cholesterol arabinogalactan anchored liposomes for asialoglycoprotein receptor mediated targeting to hepatocellular carcinoma: In silico modeling, in vitro and in vivo evaluation. International Journal of Pharmaceutics 2016, 509, (1), 149-158.

17. Marwah, M.; Magarkar, A.; Ray, D.; Aswal, V.; Bunker, A.; Nagarsenker, M. Glyceryl Monostearate: Probing the Self Assembly of a Lipid Amenable To Surface Modification for Hepatic Targeting. The Journal of Physical Chemistry C 2018, 122, (38), 22160-22169.

18. Magarkar, A.; Róg, T.; Bunker, A. A computational study suggests that replacing PEG with PMOZ may increase exposure of hydrophobic targeting moiety. European Journal of Pharmaceutical Sciences 2017, 103, 128-135.

19. Salmaso, S.; Bersani, S.; Mastrotto, F.; Tonon, G.; Schrepfer, R.; Genovese, S.; Caliceti, P. Self-assembling nanocomposites for protein delivery: Supramolecular interactions between PEG-cholane and rh-G-CSF. Journal of controlled release 2012, 162, (1), 176-184.

20. Salmaso, S.; Bersani, S.; Scomparin, A.; Balasso, A.; Brazzale, C.; Barattin, M.; Caliceti, P. A novel soluble supramolecular system for sustained rh-GH delivery. J. Controlled Release 2014, 194, 168-177.

21. Vemuri, S.; Rhodes, C. Preparation and characterization of liposomes as therapeutic delivery systems: a review. Pharm. Acta Helv. 1995, 70, (2), 95-111.

22. Mare, R.; Paolino, D.; Celia, C.; Molinaro, R.; Fresta, M.; Cosco, D. Post-insertion parameters of PEG-derivatives in phosphocholine-liposomes. International Journal of Pharmaceutics 2018, 552, (1), 414-421.

23. Sims, G. E. C.; Snape, T. J. A method for the estimation of polyethylene glycol in plasma protein fractions. Analytical Biochemistry 1980, 107, (1), 60-63.

24. Stewart, J. C. M. Colorimetric determination of phospholipids with ammonium ferrothiocyanate. Analytical Biochemistry 1980, 104, (1), 10-14.

25. Caliceti, P.; Salmaso, S.; Semenzato, A.; Carofiglio, T.; Fornasier, R.; Fermeglia, M.; Ferrone, M.; Pricl, S. Synthesis and physicochemical characterization of folate- cyclodextrin bioconjugate for active drug delivery. Bioconjug. Chem. 2003, 14, (5), 899-908. 
26. Mosmann, T. Rapid colorimetric assay for cellular growth and survival: application to proliferation and cytotoxicity assays. Journal of immunological methods 1983, 65, (1-2), 5563.

27. Gabizon, A.; Catane, R.; Uziely, B.; Kaufman, B.; Safra, T.; Cohen, R.; Martin, F.; Huang, A.; Barenholz, Y. Prolonged Circulation Time and Enhanced Accumulation in Malignant Exudates of Doxorubicin Encapsulated in Polyethylene-glycol Coated Liposomes. Cancer Research 1994, 54, (4), 987.

28. Stepniewski, M.; Pasenkiewicz-Gierula, M.; Róg, T.; Danne, R.; Orlowski, A.; Karttunen, M.; Urtti, A.; Yliperttula, M.; Vuorimaa, E.; Bunker, A. Study of PEGylated lipid layers as a model for PEGylated liposome surfaces: molecular dynamics simulation and Langmuir monolayer studies. Langmuir 2011, 27, (12), 7788-7798.

29. Magarkar, A.; Karakas, E.; Stepniewski, M.; Róg, T.; Bunker, A. Molecular Dynamics Simulation of PEGylated Bilayer Interacting with Salt Ions: A Model of the Liposome Surface in the Bloodstream. The Journal of Physical Chemistry B 2012, 116, (14), 4212-4219.

30. Lehtinen, J.; Magarkar, A.; Stepniewski, M.; Hakola, S.; Bergman, M.; Róg, T.; Yliperttula, M.; Urtti, A.; Bunker, A. Analysis of cause of failure of new targeting peptide in PEGylated liposome: molecular modeling as rational design tool for nanomedicine. Eur. $J$. Pharm. Sci. 2012, 46, (3), 121-130.

31. Lajunen, T.; Kontturi, L.-S.; Viitala, L.; Manna, M.; Cramariuc, O.; Róg, T.; Bunker, A.; Laaksonen, T.; Viitala, T.; Murtomäki, L.; Urtti, A. Indocyanine Green-Loaded Liposomes for Light-Triggered Drug Release. Molecular Pharmaceutics 2016, 13, (6), 2095-2107.

32. Lajunen, T.; Nurmi, R.; Wilbie, D.; Ruoslahti, T.; Johansson, N. G.; Korhonen, O.; Rog, T.; Bunker, A.; Ruponen, M.; Urtti, A. The effect of light sensitizer localization on the stability of indocyanine green liposomes. Journal of Controlled Release 2018, 284, 213-223.

33. Berendsen, H. J. C.; van der Spoel, D.; van Drunen, R. GROMACS: A message-passing parallel molecular dynamics implementation. Computer Physics Communications 1995, 91, (1), 43-56.

34. Hess, B.; Kutzner, C.; van der Spoel, D.; Lindahl, E. GROMACS 4: Algorithms for Highly Efficient, Load-Balanced, and Scalable Molecular Simulation. Journal of Chemical Theory and Computation 2008, 4, (3), 435-447.

35. Jorgensen, W. L.; Tirado-Rives, J. The OPLS [optimized potentials for liquid simulations] potential functions for proteins, energy minimizations for crystals of cyclic peptides and crambin. Journal of the American Chemical Society 1988, 110, (6), 1657-1666.

36. Jorgensen, W. L.; Maxwell, D. S.; Tirado-Rives, J. Development and Testing of the OPLS All-Atom Force Field on Conformational Energetics and Properties of Organic Liquids. Journal of the American Chemical Society 1996, 118, (45), 11225-11236.

37. Maciejewski, A.; Pasenkiewicz-Gierula, M.; Cramariuc, O.; Vattulainen, I.; Rog, T. Refined OPLS All-Atom Force Field for Saturated Phosphatidylcholine Bilayers at Full Hydration. The Journal of Physical Chemistry B 2014, 118, (17), 4571-4581.

38. Davis, J. H. The description of membrane lipid conformation, order and dynamics by 2H-NMR. Biochimica et Biophysica Acta (BBA) - Reviews on Biomembranes 1983, 737, (1), 117-171.

39. Humphrey, W.; Dalke, A.; Schulten, K. VMD: visual molecular dynamics. J. Mol. Graphics 1996, 14, (1), 33-38.

40. Nag, O. K.; Awasthi, V. Surface engineering of liposomes for stealth behavior. Pharmaceutics 2013, 5, (4), 542-569.

41. Soundararajan, A.; Bao, A.; Phillips, W. T.; Perez, R., 3rd; Goins, B. A. [(186)Re]Liposomal doxorubicin (Doxil): in vitro stability, pharmacokinetics, imaging and biodistribution in a head and neck squamous cell carcinoma xenograft model. Nucl Med Biol 2009, 36, (5), 515-524. 
42. Sugiyama, I.; Sadzuka, Y. Characterization of novel mixed polyethyleneglycol modified liposomes. Biological and Pharmaceutical Bulletin 2007, 30, (1), 208-211.

43. Dolor, A.; Kierstead, P.; Dai, Z.; Szoka, F. C. Sterol-modified PEG lipids: alteration of the bilayer anchoring moiety has an unexpected effect on liposome circulation. Chemical Communications 2018, 54, (84), 11949-11952.

44. Kirby, C.; Clarke, J.; Gregoriadis, G. Effect of the cholesterol content of small unilamellar liposomes on their stability in vivo and in vitro. Biochemical Journal 1980, 186, (2), 591-598.

45. Wu, E.; Jacobson, K.; Papahadjopoulos, D. Lateral diffusion in phospholipid multibilayers measured by fluorescence recovery after photobleaching. Biochemistry 1977, 16, (17), 3936-3941.

46. Deniz, A.; Sade, A.; Severcan, F.; Keskin, D.; Tezcaner, A.; Banerjee, S. Celecoxibloaded liposomes: effect of cholesterol on encapsulation and in vitro release characteristics. Bioscience reports 2010, 30, (5), 365-373.

47. Vonarbourg, A.; Passirani, C.; Saulnier, P.; Benoit, J.-P. Parameters influencing the stealthiness of colloidal drug delivery systems. Biomaterials 2006, 27, (24), 4356-4373.

48. De Gennes, P. Polymers at an interface; a simplified view. Advances in colloid and interface science 1987, 27, (3-4), 189-209.

49. Lee, H.; Larson, R. G. Adsorption of Plasma Proteins onto PEGylated Lipid Bilayers: The Effect of PEG Size and Grafting Density. Biomacromolecules 2016, 17, (5), 1757-1765.

50. Taylor, W.; Jones, R. A. Protein adsorption on well-characterized polyethylene oxide brushes on gold: Dependence on molecular weight and grafting density. Langmuir 2013, 29, (20), 6116-6122.

51. Dos Santos, N.; Allen, C.; Doppen, A.-M.; Anantha, M.; Cox, K. A. K.; Gallagher, R. C.; Karlsson, G.; Edwards, K.; Kenner, G.; Samuels, L.; Webb, M. S.; Bally, M. B. Influence of poly(ethylene glycol) grafting density and polymer length on liposomes: Relating plasma circulation lifetimes to protein binding. Biochimica et Biophysica Acta (BBA) - Biomembranes 2007, 1768, (6), 1367-1377.

52. Perry, J. L.; Reuter, K. G.; Kai, M. P.; Herlihy, K. P.; Jones, S. W.; Luft, J. C.; Napier, M.; Bear, J. E.; DeSimone, J. M. PEGylated PRINT Nanoparticles: The Impact of PEG Density on Protein Binding, Macrophage Association, Biodistribution, and Pharmacokinetics. Nano Letters 2012, 12, (10), 5304-5310.

53. Bedu-Addo, F. K.; Huang, L. Interaction of PEG-phospholipid conjugates with phospholipid: implications in liposomal drug delivery. Advanced Drug Delivery Reviews 1995, $16,(2), 235-247$.

54. Li, S.-D.; Huang, L. Pharmacokinetics and biodistribution of nanoparticles. Molecular pharmaceutics 2008, 5, (4), 496-504.

55. Danhier, F.; Feron, O.; Préat, V. To exploit the tumor microenvironment: passive and active tumor targeting of nanocarriers for anti-cancer drug delivery. Journal of controlled release 2010, 148, (2), 135-146.

56. Sawant, R. R.; Torchilin, V. P. Challenges in development of targeted liposomal therapeutics. The AAPS journal 2012, 14, (2), 303-315.

57. Caracciolo, G.; Callipo, L.; De Sanctis, S. C.; Cavaliere, C.; Pozzi, D.; Laganà, A. Surface adsorption of protein corona controls the cell internalization mechanism of DC-CholDOPE/DNA lipoplexes in serum. Biochimica et Biophysica Acta (BBA)-Biomembranes 2010, 1798, (3), 536-543.

58. Kari, O. K.; Rojalin, T.; Salmaso, S.; Barattin, M.; Jarva, H.; Meri, S.; Yliperttula, M.; Viitala, T.; Urtti, A. Multi-parametric surface plasmon resonance platform for studying liposome-serum interactions and protein corona formation. Drug delivery and translational research 2017, 7, (2), 228-240. 
59. Barattin, M.; Mattarei, A.; Balasso, A.; Paradisi, C.; Cantù, L.; Del Favero, E.; Viitala, T.; Mastrotto, F.; Caliceti, P.; Salmaso, S. pH-controlled liposomes for enhanced cell penetration in tumor environment. ACS applied materials \& interfaces 2018.

60. Mourtas, S.; Michanetzis, G. P. A. K.; Missirlis, Y. F.; Antimisiaris, S. G. Haemolytic Activity of Liposomes: Effect of Vesicle Size, Lipid Concentration and Polyethylene GlycolLipid or Arsonolipid Incorporation. Journal of Biomedical Nanotechnology 2009, 5, (4), 409415.

61. Nie, Y.; Ji, L.; Ding, H.; Xie, L.; Li, L.; He, B.; Wu, Y.; Gu, Z. Cholesterol derivatives based charged liposomes for doxorubicin delivery: preparation, in vitro and in vivo characterization. Theranostics 2012, 2, (11), 1092-1103.

62. Olson, F.; Mayhew, E.; Maslow, D.; Rustum, Y.; Szoka, F. Characterization, toxicity and therapeutic efficacy of adrïamycin encapsulated in liposomes. European Journal of Cancer and Clinical Oncology 1982, 18, (2), 167-176.

63. Parnham, M. J.; Wetzig, H. Toxicity screening of liposomes. Chemistry and Physics of Lipids 1993, 64, (1), 263-274.

64. Cole, A. J.; David, A. E.; Wang, J.; Galbán, C. J.; Yang, V. C. Magnetic brain tumor targeting and biodistribution of long-circulating PEG-modified, cross-linked starch-coated iron oxide nanoparticles. Biomaterials 2011, 32, (26), 6291-6301.

65. Golkar, N.; Tamaddon, A. M.; Samani, S. M. Effect of lipid composition on incorporation of trastuzumab-PEG-lipid into nanoliposomes by post-insertion method: physicochemical and cellular characterization. Journal of Liposome Research 2016, 26, (2), 113-125.

66. Polyak, D.; Krivitsky, A.; Scomparin, A.; Eliyahu, S.; Kalinski, H.; Avkin-Nachum, S.; Satchi-Fainaro, R. Systemic delivery of siRNA by aminated poly(alpha)glutamate for the treatment of solid tumors. Journal of controlled release : official journal of the Controlled Release Society 2017, 257, 132-143.

67. Tanaka, T.; Legat, A.; Adam, E.; Steuve, J.; Gatot, J. S.; Vandenbranden, M.; Ulianov, L.; Lonez, C.; Ruysschaert, J. M.; Muraille, E.; Tuynder, M.; Goldman, M.; Jacquet, A. DiC14amidine cationic liposomes stimulate myeloid dendritic cells through Toll-like receptor 4. European journal of immunology 2008, 38, (5), 1351-7.

68. Fröhlich, E. The role of surface charge in cellular uptake and cytotoxicity of medical nanoparticles. International journal of nanomedicine 2012, 7, 5577.

69. Pozzi, D.; Colapicchioni, V.; Caracciolo, G.; Piovesana, S.; Capriotti, A. L.; Palchetti, S.; De Grossi, S.; Riccioli, A.; Amenitsch, H.; Laganà, A. Effect of polyethyleneglycol (PEG) chain length on the bio-nano-interactions between PEGylated lipid nanoparticles and biological fluids: from nanostructure to uptake in cancer cells. Nanoscale 2014, 6, (5), 2782-2792.

70. Neoh, K. G.; Kang, E. T. Functionalization of inorganic nanoparticles with polymers for stealth biomedical applications. Polymer Chemistry 2011, 2, (4), 747-759.

71. Murari, R.; Murari, M. P.; Baumann, W. J. Sterol orientations in phosphatidylcholine liposomes as determined by deuterium NMR. Biochemistry 1986, 25, (5), 1062-1067.

72. Matsumura, Y.; Maeda, H. A new concept for macromolecular therapeutics in cancer chemotherapy: mechanism of tumoritropic accumulation of proteins and the antitumor agent smancs. Cancer research 1986, 46, (12 Part 1), 6387-6392.

73. Amantea, M. A.; Forrest, A.; Northfelt, D. W.; Mamelok, R. Population pharmacokinetics and pharmacodynamics of pegylated-liposomal doxorubicin in patients with AIDS-related Kaposi's sarcoma. Clinical Pharmacology \& Therapeutics 1997, 61, (3), 301311.

74. Wagener, K.; Worm, M.; Pektor, S.; Schinnerer, M.; Thiermann, R.; Miederer, M.; Frey, H.; Rösch, F. Comparison of Linear and Hyperbranched Polyether Lipids for Liposome Shielding by $18 \mathrm{~F}-$ Radiolabeling and Positron Emission Tomography. Biomacromolecules 2018, 19, (7), 2506-2516. 
75. de Gennes, P. G. Conformations of Polymers Attached to an Interface. Macromolecules 1980, 13, (5), 1069-1075.

76. Torchilin, V. P. Polymer-coated long-circulating microparticulate pharmaceuticals. Journal of Microencapsulation 1998, 15, (1), 1-19.

77. Cunningham, C. M.; Kingzette, M.; Richards, R. L.; Alving, C. R.; Lint, T. F.; Gewurz, H. Activation of Human Complement by Liposomes: A Model for Membrane Activation of the Alternative Pathway. The Journal of Immunology 1979, 122, (4), 1237.

78. Romberg, B.; Oussoren, C.; Snel, C. J.; Carstens, M. G.; Hennink, W. E.; Storm, G. Pharmacokinetics of poly(hydroxyethyl-1-asparagine)-coated liposomes is superior over that of PEG-coated liposomes at low lipid dose and upon repeated administration. Biochimica et Biophysica Acta (BBA) - Biomembranes 2007, 1768, (3), 737-743. 


\section{For Table of Contents Use Only}

In vitro and in vivo behavior of liposomes decorated with PEGs with different chemical features.

Francesca Mastrotto $^{1}$, Chiara Brazzale ${ }^{1}$, Federica Bellato ${ }^{1}$, Sara De Martin ${ }^{1}$, Guillaume Grange $^{2}$, Mohamad Mahmoudzadeh $^{2}$, Aniket Magarkar ${ }^{3}$, Alex Bunker $^{2}$, Stefano Salmaso ${ }^{*}$, Paolo Caliceti ${ }^{1}$

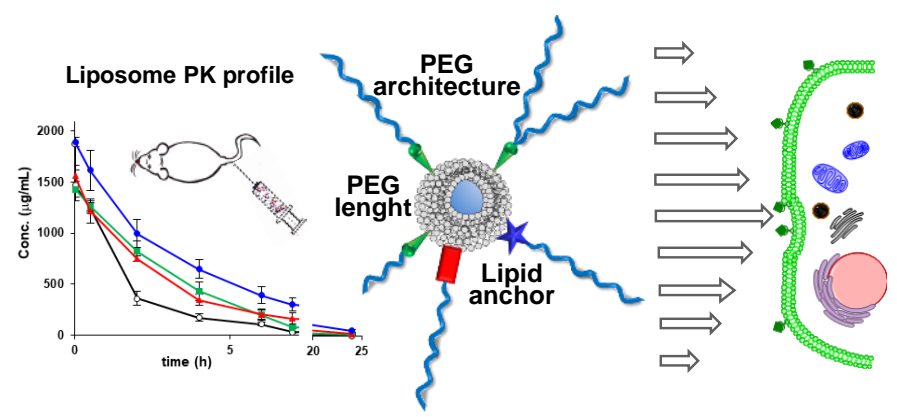

According to their chemical structure, PEGylating agents for liposomes coating possess different "stealthing" performance, which reflects on unlike liposome cell interaction and pharmacokinetic profile. The effect of PEG length and architecture and the lipid anchor on biopharmaceutical and pharmacokinetic profile of PEGylated liposomes has been elucidated. 